DESY 93-083

hep-ph/9307274

\title{
Equations of Motion for Effective Lagrangians and Penguins in Rare B-Decays
}

\author{
H. Simma \\ Deutsches Elektronen-Synchrotron DESY, Hamburg
}

\begin{abstract}
We study the application of the classical equations of motion (EOM) within the framework of an effective low-energy Lagrangian treated at the loop level. Gaugefixing and ghost terms, which enter naturally in the EOM, are found to lead to no physical effects - neither through operator mixing nor in matrix elements. Beyond first order in the effective interactions, contact terms have to be included when reducing the effective Lagrangian and we present an explicit procedure to construct them. Applied to (hadronic) rare B-decays, the EOM drastically simplify the effective Lagrangian and its matching to the underlying theory, and certain cancellations of large (logarithmic) contributions become more transparent. Finally, we discuss details of the 'matching' of the effective Lagrangian, which may be helpful in incorporating short distance QCD corrections in further phenomenological studies.
\end{abstract}




\section{Introduction}

Processes in which the external momenta and masses are much smaller than the mass scale of the particles that mediate the interactions, are most conveniently described in the framework of an effective field theory [1]. There, the heavy particles are integrated out and their relevant effects are summarized in a concise way by local operators in an effective Lagrangian. Moreover, short-distance (QCD) corrections can be included in a systematic way by means of the renormalization group. Typical phenomenological applications of effective theories are quantitative predictions for low-energy processes on the basis of a given theory (e.g. the standard model) or when experimental data is analyzed in order to obtain bounds or hints for yet unknown 'new physics' (e.g. through anomalous gauge-boson couplings).

The complete set of linearly independent effective operators for certain physical processes usually contains many operators which are formally related by the classical equations of motion (EOM). It is desirable to exploit these relations among the various operators to simplify the expression for a given effective Lagrangiand and at any stage of the calculation (e.g. after integrating out heavy particles and before performing the renormalization group evolution). To this end, we investigate in this paper the explicit form of these relations and their preservation by renormalization effects.

Of course, at tree (i.e. classical) level, on-shell matrix elements of an effective operator may be simplified by using the classical EOM, which include the interactions from the usual, e.g. $Q C D \times Q E D$, Lagrangian (to account for the fact that internal lines entering in the effective operator may actually be off shell). When working at higher order in the effective interactions, a more careful formulation of the EOM, which will also depend on the effective interactions themselves, is necessary. (Typically, an effective Lagrangian is applied only at first order, but one may think of processes, like $K^{o}-\bar{K}^{o}$ or $B^{o}-\bar{B}^{o}$ mixing, which arise only at second order in the effective weak interactions.)

At the loop level also renormalization effects have to be taken into account, and they are, in fact, crucial for assessing the applicability of the EOM before the renormalization group (RG) evolution. The renormalization of composite operators is well-known and has been studied in great detail [3, 4, 5, 6]; however, in phenomenological work the issue of the EOM is usually either not discussed in a coherent way or the general results are

\footnotetext{
${ }^{1}$ i.e. a linear combination of effective operators with fixed coefficients. Note the difference to the question of simply reducing the set of operators to describe general on-shell amplitudes. For instance, it has been stressed [2] that operators containing covariant derivatives, $\not D \Psi, D_{\mu} G^{\mu \nu}$, etc., can be dropped from the complete set of operators. However, the explicit form of the relation between the operators is not specified; rather their coefficients are to be determined by a complicated matching procedure where many additional 1PR graphs must be evaluated.
} 
not exploited.

A complication, which is sometimes overlooked, arises from the fact that the appropriate Lagrangian for the quantized theory contains gauge-fixing terms and FaddeevPopov ghosts. A priori, these terms are to be taken into account in the EOM and they can, in principle, contribute to physical processes through mixing with physical operators and/or through non-vanishing matrix elements at the loop level. The crucial point is that the additional gauge-fixing and ghost terms enter the EOM in a combination, which is the BRS variation of some other operator; then, using standard arguments [5, 7], we find that these additional terms do not affect the matrix elements for physical processes. Throughout this paper we shall use the convention that the "classical EOM' include possible gauge-fixing and ghost contributions — in contrast to the 'naive classical EOM' without these terms.

While our discussion of the EOM can be useful in various other phenomenological studies (for instance, of anomalous gauge-boson couplings), we focus our attention in the second part of this paper on hadronic rare B-decays . In particular, we consider here flavour changing neutral $b \rightarrow s$ currents which arise from loop diagrams, so called 'penguins', with internal $u, c$ or top quark. The corresponding tree diagrams - if at all present - are highly CKM suppressed.

Penguin diagrams do not only induce interesting exclusive decays, like $B \rightarrow K^{*} \gamma$ [8], but also contribute considerably to the inclusive hadronic branching ratio for $b \rightarrow$ $s+$ no charm (via $b \rightarrow s q \bar{q}, b \rightarrow s g g$, etc. [9]), and they can generate CP violating asymmetries in charged B-decays [10]. In various of these processes, one finds a remarkable cancellation [11] of large - since only weakly GIM suppressed - contributions with logarithmic dependence on the internal mass. We demonstrate how this cancellation, which in fact has been missed in the pioneering works [9, 12, is made transparent by the EOM.

The EOM are particularly interesting for calculations of short-distance QCD effects (which lead to sizable corrections in the branching ratios [13] and asymmetries [14, 15]). In the abundant literature on such calculations for K- and B-meson decays, essentially two approaches are to be found: Many authors perform the RG evolution with an effective Lagrangian not reduced by the EOM (see for instance refs. [16, 17, 18]). Of course, this is a rather tedious procedure because the corresponding anomalous dimension matrix is large. In some cases it has been observed that the final result for the process considered is independent of whether the EOM are applied before or after performing the RG evolution. While in ref. [17] this fact has been interpreted as a nontrivial result of the calculation, the authors of ref. [16] suspected that this is not an accidental fact, but did not give a general argument. 
Other authors, for instance, Grinstein et al. [19] and Buras et al. [20, employ the naive classical EOM to simplify the operator basis right from the beginning. The applicability of these EOM is either not discussed explicitly or assumed on the basis

of a formal proof by Politzer [21] (which by a close inspection actually leads to the classical EOM including the gauge-fixing and ghost terms). We shall clarify this point by demonstrating the appearance of the gauge-fixing and ghost contributions in terms of diagrams and by investigating their renormalization.

Finally, we would like to discuss some details concerning the 'matching' of the full theory with the effective Lagrangian (reduced by the EOM), and the recovery of the momentum dependent and absorptive parts of the amplitudes in the effective theory at leading and next-to-leading log precision. These issues are often somewhat obscured in the literature by other technical details related to the actual calculation of the RG evolution.

The remainder of this paper is organized as follows: In section 2 we review the proof of the EOM and verify the absence of physical effects from the gauge-fixing and ghost terms. We then discuss the appropriate form of the EOM when working at higher order in the effective Lagrangian. In section 3 we exploit the EOM to reduce the effective penguin operators in $b \rightarrow s$ transitions, thereby taking inventory of the coefficients with logarithmic mass dependence, and we describe the 'matching' of the effective Lagrangian. The discussion in section 4 contains some clarifying remarks, and additional details, which may be helpful for further applications, are collected in the appendices.

\section{Equations of motion}

\subsection{General framework}

The Lagrangian of an effective theory [1] consists of two parts:

$$
\mathcal{L}=\mathcal{L}_{\mathrm{o}}+\mathcal{L}_{\text {eff }}
$$

$\mathcal{L}_{\mathrm{o}}$ is renormalizable by power counting and describes those interactions of the particles which, in principle, are to be taken into account at arbitrary order in perturbation theory. Of course, for a consistent quantization $\mathcal{L}_{\mathrm{o}}$ has to include terms for the gaugefixing and Faddeev-Popov ghosts. The effective Lagrangian, $\mathcal{L}_{\text {eff }}$, is a linear combination of higher dimensional local operators representing the effective interactions. They have dimensionful couplings and are to be treated only up to a certain order, usually the first one. The effective interactions may be thought as being induced by heavy particles of an underlying more fundamental theory. A typical example is $\mathcal{L}_{\text {eff }}$ arising from effective 
weak interactions (induced by virtual W's and top quarks) with $\mathcal{L}_{\mathrm{o}}=\mathcal{L}_{\mathrm{QCD}}$ being the usual QCD Lagrangian (neglecting QED interactions for simplicity).

In order to regularize UV divergences in calculations beyond the tree level we will always assume dimensional regularization. The composite operators, $O_{i}(y)$, in $\mathcal{L}_{\text {eff }}$ are defined [3, 7] by their Green's functions $\left\langle T O_{i}(y) X\left(x_{1}, \ldots\right)\right\rangle$, where $T$ indicates timeordering and the fields are interacting with respect to $\mathcal{L}_{\mathrm{o}}$. Physical matrix elements or operator identities are obtained by LSZ reduction, and the Green's functions themselves are defined perturbatively by (regularized) Feynman graphs. In the following, $X$ will always denote a product of elementary fields at different points

$$
X\left(x_{1}, \ldots\right) \equiv \Phi\left(x_{1}\right) \ldots A_{\mu_{1}}^{a_{1}}\left(x_{k+1}\right) \ldots \bar{\Psi}\left(x_{m}\right) \ldots \Psi\left(x_{n}\right) \ldots .
$$

For fixed values of the regularization parameter $\epsilon$ and of the renormalization scale $\mu$, the renormalized effective theory is specified by three elements: (i) The Lagrangian, $\mathcal{L}(g, \cdots ; \Phi)$, as a function of the fields and parameters (like couplings and masses), (ii) the values $g^{R}, \ldots$ for these parameters, and (iii) the counter term Lagrangian, $\mathcal{L}^{\text {ct }}$ - or, equivalently, a renormalization prescription to determine $\mathcal{L}^{\text {ct }}$ from $\mathcal{L}$. We call $\mathcal{L}^{\text {basic }}(\Phi) \equiv \mathcal{L}\left(\mu^{\epsilon} g^{R}, \ldots ; \Phi\right)$ the 'basic' part of the 'renormalized' Lagrangian $\mathcal{L}^{R}(\Phi) \equiv$ $\mathcal{L}^{\text {basic }}(\Phi)+\mathcal{L}^{\text {ct }}\left(\mu^{\epsilon} g^{R}, \ldots, \epsilon ; \Phi\right)$, where the usual powers of $\mu^{\epsilon}$ keep the dimensions of the $g^{R}$ independent of $\epsilon$. In the following, we will always assume minimal (or $\overline{m s}$ ) subtraction for the renormalization prescription.

Renormalized Green's functions are calculated by using $\mathcal{L}^{R}$ and remain finite for $\epsilon \longrightarrow 0$ due to suitable counter terms in $\mathcal{L}^{\text {ct }}$. While all counter terms needed for Green's functions of elementary fields are proportional to terms in $\mathcal{L}_{\mathrm{o}}$ (multiplicative renormalization of $\mathcal{L}_{\mathrm{o}}$ ), the renormalization of insertions of composite operators requires additional subtractions proportional to other composite operators. Since $\mathcal{L}_{\text {eff }}$ is to be kept only up to a finite order, only a finite number of such counterterms is needed; in this sense $\mathcal{L}_{\mathrm{o}}+\mathcal{L}_{\text {eff }}$ is renormalizable. To render the effective operators multiplicatively renormalizable, one includes a priori all interactions in $\mathcal{L}_{\text {eff }}$ that may appear as counterterms.

The couplings of $\mathcal{L}_{\text {eff }}$, i.e. the coefficient functions of the operators, are determined by 'matching' on-shell amplitudes either with a more fundamental theory or with experimental data. By this procedure no distinction is possible between effective theories which yield the same on-shell amplitudes. Henceforth, different Lagrangians yielding the same on-shell amplitudes will be called 'on-shell equivalent'. An 'on-shell effective theory' [1] can be viewed as a class of on-shell equivalent effective theories.

If two (effective) theories, renormalized at any scale $\mu$, are on-shell equivalent, then the corresponding 'bare' theoriest are also on-shell equivalent, and vice versa. Since

\footnotetext{
${ }^{2}$ with bare parameters, $g_{0}(\epsilon), \ldots$, which are divergent functions of $\epsilon$, such that the regularized bare
} 
the renormalization group describes the relation between the parameters of the various renormalized theories corresponding to the same bare theory but different values of $\mu$, the RG evolution preserves the on-shell equivalence of effective Lagrangians. Consequently, one can simplify the expression for $\mathcal{L}_{\text {eff }}$ already before the $\mathrm{RG}$ evolution by replacing $\mathcal{L}_{\text {eff }}$ by an on-shell equivalent 'reduced' effective Lagrangian.

To study how the equations of motion can be exploited in order to obtain on-shell equivalent effective Lagrangians, we consider a generic effective operator $O_{\mathrm{EOM}}$, which contains the fields in a combination that vanishes by the classical EOM derived from $\mathcal{L}_{\mathrm{o}}$ :

$$
O_{\mathrm{EOM}}(z)=Q(z) \cdot\left(\frac{\delta \mathcal{L}_{\mathrm{o}}}{\delta \Phi(z)}-\partial_{\mu} \frac{\delta \mathcal{L}_{\mathrm{o}}}{\delta \partial_{\mu} \Phi(z)}\right),
$$

where $\Phi$ represents one of the fields from $\mathcal{L}_{\mathrm{o}}$, and $Q$ is a monomial in any of the fields and their derivatives (at the same point). Of course, we exclude the case $Q=\Phi$, since then $O_{\mathrm{EOM}}$ would correspond to an operator in $\mathcal{L}_{\mathrm{o}}$ and, in particular, would contain the kinetic energy term. For Green's functions with an insertion of $O_{\mathrm{EOM}}$ one finds [3, 4] (after integration over $z$ to avoid derivatives of Dirac delta functions)

$$
\begin{aligned}
& \int\left\langle T O_{\mathrm{EOM}}(z) O_{1}\left(y_{1}\right) \ldots O_{n}\left(y_{n}\right) X\left(x_{1}, \ldots\right)\right\rangle d z= \\
& \quad i \int\left\langle T \sum_{\Lambda} \Lambda[Q(z)] \frac{\delta}{\delta \Lambda[\Phi(z)]}\left(O_{1}\left(y_{1}\right) \ldots O_{n}\left(y_{n}\right) X\left(x_{1}, \ldots\right)\right)\right\rangle d z
\end{aligned}
$$

where $\Lambda[f] \equiv f, \partial_{\mu} f, \partial_{\mu} \partial_{\nu} f, \ldots$, denotes the various derivatives (including the fields themselves) that may enter in the composite operators $O_{i}$.

For bare Green's functions eq. (2) is readily derived by inspection of the contributing Feynman diagrams [see subsection 2.2 for an illustration]. The r.h.s. of (2) consists of contact terms which contribute only when $z$ coincides with one of the $x_{1}, \ldots$ or $y_{1}, \ldots, y_{n}$. They originate from diagrams where the inverse propagator in $O_{\mathrm{EOM}}$ cancels either an external line from one of the elementary fields in $X\left(x_{1}, \ldots\right)$, or an internal line ending up in a vertex generated by one of the other composite operators $O_{1}\left(y_{1}\right) \ldots O_{n}\left(y_{n}\right)$, respectively.

Equation (2) is also valid for the renormalized Green's functions; the derivatives $\delta / \delta \Phi$ etc. in (1) and (2) are then to be taken with respect to the renormalized fields and $\mathcal{L}_{\mathrm{o}}$ refers only to the basic part of the Lagrangian. The renormalized version of (2) is shown either in terms of diagrams [4], or using the multiplicative renormalization of the composite operators by induction in the number of loops and insertions.

Green's functions, calculated from the bare Lagrangian $\mathcal{L}^{\text {bare }}(\Phi) \equiv \mathcal{L}\left(g_{0}(\epsilon), \ldots ; \Phi\right)$, differ from the renormalized ones only by field-renormalization factors. 


\section{$2.2 \quad$ EOM at first order in $\mathcal{L}_{\text {eff }}$}

In many applications of effective theories the interactions from the effective Lagrangian are to be treated only at first order. Therefore, we restrict ourselves with the exception of subsection 2.5 to this case. We write

$$
\mathcal{L}_{\text {eff }}^{R}=g_{\text {eff }} \sum c_{i} O_{i}+\text { counterterms }
$$

where $g_{\text {eff }}$ collectively denotes the dimensionful couplings (for instance, $g_{\text {eff }}=G_{F}$ in the case of effective weak interactions). All quantities to be considered are at most linear in $g_{\text {eff }}$ or, equivalently, in the coefficient functions $c_{i}$.

In particular, only Green's functions with single insertions of the composite operators from $\mathcal{L}_{\text {eff }}$ are needed, and (2) becomes particularly simple

$$
\begin{aligned}
& \int\left\langle T O_{\mathrm{EOM}}(z) \Phi\left(x_{1}\right) \ldots \Phi\left(x_{k}\right) \hat{X}\left(x_{k+1}, \ldots\right)\right\rangle d z= \\
& \quad i \sum_{j=1}^{k}\left\langle T \Phi\left(x_{1}\right) \ldots \Phi\left(x_{j-1}\right) Q\left(x_{j}\right) \Phi\left(x_{j+1}\right) \ldots \Phi\left(x_{k}\right) \hat{X}\left(x_{k+1}, \ldots\right)\right\rangle,
\end{aligned}
$$

where $\Phi$ denotes the field to which the variation in (11) refers, and $\hat{X}$ is a product of elementary fields containing no $\Phi$ 's. The contact terms on the r.h.s. do not survive the LSZ reduction and, therefore, do not contribute to matrix elements of physical processes (see e.g. Joglekar in ref. [5], and [21] for a discussion of this issue beyond the parton level). Hence, any multiple of $O_{\mathrm{EOM}}$ can simply be dropped from $\mathcal{L}_{\text {eff }}$.

Inspecting the reasoning by which (田) is derived for the renormalized Green's functions, one notes that the counterterms for the renormalization of $O_{\mathrm{EOM}}$ are themselves proportional to operators which vanish by the classical EOM. Of course, the counterterms are in general not proportional to $O_{\mathrm{EOM}}$ itself, but in fact related to counterterms for $Q$. For instance, in $\Phi^{3}$ theory, $O_{\mathrm{EOM}}=\Phi^{3}\left(\partial^{2} \Phi+m^{2} \Phi+g \Phi^{2} / 2\right)$ requires counterterms proportional to $\Phi^{2}\left(\partial^{2} \Phi+m^{2} \Phi+g \Phi^{2} / 2\right)$ etc.

The derivation of (2) or (4) in the path integral representation by a change of variables is not very intuitive, and might have even been misleading in cases when ref. [21] is quoted in order to justify the naive classical EOM for the gluons (without gauge-fixing and ghost terms). To illustrate the derivation in terms of the contributing Feynman diagrams we consider here the EOM for the gauge-boson (gluon) field strength

$$
D_{\mu} G_{a}^{\mu \nu} \equiv\left(\partial_{\mu} \delta_{a b}-g_{s} f_{a b c} A_{\mu}^{c}\right) G_{b}^{\mu \nu}=\left(J_{\bar{q} q}+J_{\mathrm{gf}}+J_{\mathrm{FP}}\right)_{a}^{\nu}
$$

While in the naive classical EOM only the quark contribution

$$
\left(J_{\bar{q} q}\right)_{a}^{\nu}=-g_{s} \sum_{\text {quarks }} \bar{q} \gamma^{\nu} \frac{\lambda^{a}}{2} q
$$


is present, the two other terms on the r.h.s., $J_{\mathrm{gf}}$ and $J_{\mathrm{FP}}$, arise in an unambiguous way from the gauge-fixing and ghost terms in the Lagrangian.

In a covariant gauge (for expressions in a background gauge see appendix A) the gauge-fixing and Faddeev-Popov parts of the Lagrangian are given by

$$
\mathcal{L}_{\mathrm{gf}}=-\frac{1}{2 \xi}(\partial A)^{2}, \quad \text { and } \quad \mathcal{L}_{\mathrm{FP}}=-\left(\partial_{\mu} \bar{\eta}_{a}\right) D_{a b}^{\mu} \eta_{b}
$$

respectively, and the resulting currents on the r.h.s. of (5) are

$$
\left(J_{\mathrm{gf}}\right)_{a}^{\nu}=-\frac{1}{\xi} \partial^{\nu} \partial_{\mu} A_{a}^{\mu}, \quad \text { and } \quad\left(J_{\mathrm{FP}}\right)_{a}^{\nu}=-g_{s} f_{a b c}\left(\partial^{\nu} \bar{\eta}_{b}\right) \eta_{c}
$$

A generic operator vanishing by the classical EOM (5) has the form

$$
O_{\mathrm{EOM}} \equiv Q \cdot D_{\mu} G^{\mu \nu}-O_{\bar{q} q}-O_{\mathrm{FP}}-O_{\mathrm{gf}}
$$

where $Q$ denotes an arbitrary combination of further fields at the same point and $O_{\bar{q} q} \equiv$ $Q \cdot J_{\bar{q} q}$, etc. $D_{\mu} G^{\mu \nu}$ consists of a one-, a two-, and a three-gluon piece. The one-gluon piece, $\partial_{\mu} \partial^{\mu} A^{\nu}-\partial^{\nu} \partial_{\mu} A^{\mu}$, can be written in the form $O_{0}+J_{\text {gf }}$. Then, $J_{\text {gf }}$ cancels against the last term on the r.h.s. of (9), and the remainder $O_{0}$ is proportional to the inverse gluon propagator $\left(q^{2} g_{\mu \nu}-\frac{\xi-1}{\xi} q_{\mu} q_{\nu}\right.$ in momentum space).

To illustrate (4) for $O_{\mathrm{EOM}}$ of eq. (9), we investigate first the (regularized) diagrams which have a vertex from $O_{0}$. The external legs of the diagrams are given by the elementary fields in (4) [with $\left.\Phi \equiv A_{a}^{\mu}\right]$ which are on shell for physical matrix elements. If the gluon field in $O_{0}$ is contracted with an external gluon, the diagram contributes to the contact terms on the r.h.s. of (幽). The inverse propagator in $O_{0}$ acts on the external leg and yields zero for on-shell matrix elements. Possible diagrams in which the gluon field in $O_{0}$ is contracted with a gluon in $Q$ at the same point vanish in dimensional regularization. In all other diagrams with a vertex from $O_{0}$ (working at first order in $\mathcal{L}_{\text {eff }}$ !), the gluon propagator attached to $O_{0}$ ends in an usual QCD-vertex, $V$, and is effectively canceled by the inverse propagator in $O_{0}$.

These diagrams, with a vertex from $O_{0}$ next to a QCD-vertex $V$, are in one-to-one correspondence to diagrams with one of the remaining vertices from $O_{\mathrm{EOM}}$ : A diagram with $V$ being a three- or a four-gluon vertex cancels with the corresponding diagram containing the two- or the three-gluon piece of $D_{\mu} G^{\mu \nu}$, respectively (see fig. 1). Finally, if $V$ is a quark-gluon or a ghost-gluon vertex, then the diagram is canceled by the corresponding one with a vertex from $O_{\bar{q} q}$ or $O_{\mathrm{FP}}$, respectively.

\footnotetext{
3 The vanishing of these 'tadpole'-like diagrams is a crucial property of dimensional regularization needed here; otherwise, additional vacuum subtractions are necessary.
} 
Similar considerations can be readily carried out for an operator which vanishes by the classical EOM for the fermions (quarks),

$$
i \not D \Psi \equiv i\left(\not \partial-i g_{s} \not A^{a} \frac{\lambda^{a}}{2}\right) \Psi=m \Psi,
$$

or for the case of scalars (see also [7]) and ghosts.

The main technical complication in proving the renormalized version of (2) or (4) by means of diagrams arises from the fact that bare graphs which are in one-to-one correspondence (in the sense that they cancel each other) can have a different 1PI structure. Thus, their 'forests', which prescribe the renormalization, are not in oneto-one correspondence, and it has to be shown that one can remove recursively those forests which are not in one-to-one correspondence [4].

\subsection{Terms from gauge-fixing and ghosts}

To study the relevance of the terms $J_{\mathrm{gf}}+J_{\mathrm{FP}}$ in the EOM for the gauge-boson field strength [see the r.h.s. of eq (9)], it is convenient to define two sets of operators:

$\mathcal{O}_{\mathrm{E}}$ : Linear combinations of operators which vanish by the classical EOM derived from (the basic part of) $\mathcal{L}_{\mathrm{o}}$. Of course, terms from gauge-fixing and ghosts in $\mathcal{L}_{\mathrm{o}}$, and the EOM for the ghosts themselves [see (A.4)] are to be taken into account as well.

$\mathcal{O}_{\mathrm{B}}$ : Linear combinations of operators which are the BRS variation of some other operator.

The key observation for the discussion of the gauge-fixing and ghost terms is the fact that $O_{\text {gf }}+O_{\mathrm{FP}}$ belongs to $\mathcal{O}_{\mathrm{B}}$ provided that $Q_{\nu}^{a}$ varies under BRS transformations as

$$
\delta Q_{\nu}^{a}=g_{s} f_{a b c} Q_{\nu}^{b} \eta^{c}
$$

(with the shorthand notation $\delta \equiv \delta_{\mathrm{BRS}} / \delta \omega$, where $\delta \omega$ is the infinitesimal parameter of the transformation). Then,

$$
O_{\mathrm{FP}}+O_{\mathrm{gf}}=\delta\left(-Q_{\mu}^{a} \partial^{\mu} \bar{\eta}_{a}\right)
$$

Obviously, eq. (11) holds if $Q_{\nu}^{a} D_{\mu} G_{a}^{\mu \nu}$ is gauge-invariant and contains no ghosts. Typical examples for dimension six operators are $Q_{\nu}^{a}=D^{\mu} G_{\mu \nu}^{a}$ or $Q_{\nu}^{a}=\bar{\Psi} \gamma_{\nu} \lambda^{a} \Psi$, where $\Psi$ may be left- or right-handed and a vector in flavour space. After applying the EOM twice to $\left(D^{\mu} G_{\mu \nu}^{a}\right)\left(D_{\lambda} G_{a}^{\lambda \nu}\right)$ one arrives at $Q_{\nu}^{a}$ containing ghosts in the combination $Q_{\nu}^{a}=\left(J_{\mathrm{gf}}+J_{\mathrm{FP}}\right)_{\nu}^{a}$. In this case an operator from $\mathcal{O}_{\mathrm{E}}$, which vanishes by the EOM for the ghosts, has to be added on the r.h.s. of (11) and (12).

To see that an $O_{\mathrm{BRS}} \in \mathcal{O}_{\mathrm{B}}$ does not contribute to physical (on shell) matrix elements, one writes $O_{\mathrm{BRS}}=\delta \hat{O}$ and recalls (e.g. [7])

$$
\left\langle T \delta \hat{O}(y) X\left(x_{1}, \ldots\right)\right\rangle=-\left\langle T \hat{O}(y) \delta X\left(x_{1}, \ldots\right)\right\rangle .
$$


The r.h.s. vanishes after LSZ reduction, because $\delta X$ contains composite fields which do not lead to a physical particle pole.

It is illustrating to compare the physical matrix elements of $O_{\mathrm{gf}}$, for instance for $Q_{\nu}^{a}=\bar{\Psi} \gamma_{\nu} T_{a} \Psi$, with those of $J_{\text {gf }}^{\nu a}$. The fact that matrix elements of $J_{\text {gf }}^{\nu a} \quad\left[=\delta\left(\partial^{\nu} \bar{\eta}^{a}\right) \in \mathcal{O}_{\mathrm{B}}\right]$ are zero is just the familiar current conservation: The amplitude for " $g * \rightarrow$ physical onshell particles" vanishes when one substitutes the polarization vector of the gluon $g^{*}$ by its momentum (which can be on or off shell). On the other hand, $O_{\text {gf }}$ alone, which is not in $\mathcal{O}_{\mathrm{B}}$, has non-vanishing matrix elements. This is readily understood for diagrams in which the outgoing gluon line $g^{*}$ from $J_{\text {gf }}$ closes a loop (e.g. in fig. 2a): At the Ogf-vertex the gluon propagator is indeed contracted by its momentum; however, the diagram corresponds to an amplitude where the $g^{*}$ 'decays' into an off-shell particle that again enters the $O_{\text {gf }}$-vertex. The overall contribution of these diagrams is canceled by the matrix element of $O_{\mathrm{FP}}$ in fig. $2 \mathrm{~b}$ (this can be verified directly by manipulation of the corresponding diagrams [22]).

As to the renormalization of operators from $\mathcal{O}_{\mathrm{B}}$, one considers an $O_{\mathrm{BRS}} \equiv \delta \hat{O} \in \mathcal{O}_{\mathrm{B}}$ and counterterms $\hat{C}$, which render all Green's functions of $\hat{O}$ with elementary fields finite. Then, the Green's functions

$$
\left\langle T\left(O_{\mathrm{BRS}}+\delta \hat{C}\right)(y) \cdot X\left(x_{1}, \ldots\right)\right\rangle^{R_{\mathrm{o}}}=-\left\langle T(\hat{O}+\hat{C})(y) \cdot \delta X\left(x_{1}, \ldots\right)\right\rangle^{R_{\mathrm{o}}},
$$

where $R_{\mathrm{o}}$ indicates counterterms from $\mathcal{L}_{\mathrm{o}}$, can only be divergent if $y$ coincides with one of the $x_{i}$ (related with a composite field in $\delta X$ ). Hence, only some operators from $\mathcal{O}_{\mathrm{E}}$, together with $\delta \hat{C} \in \mathcal{O}_{\mathrm{B}}$, are necessary as counterterms for $O_{\mathrm{BRS}}$.

\subsection{Reduction and RG evolution at first order in $\mathcal{L}_{\text {eff }}$}

The stability of $\mathcal{O}_{\mathrm{E}}$ (respectively $\mathcal{O}_{\mathrm{E}} \oplus \mathcal{O}_{\mathrm{B}}$ ) under renormalization, together with the fact that these operators have vanishing on-shell matrix elements, implies that one may use the (naive) classical EOM to reduce the basic part of an effective Lagrangian already before the RG evolution: If the basic parts of two Lagrangians, renormalized at some scale $\mu$, differ only by operators which are in $\mathcal{O}_{\mathrm{E}}\left(\mathcal{O}_{\mathrm{E}} \oplus \mathcal{O}_{\mathrm{B}}\right)$, then the corresponding renormalized theories are on-shell equivalent, and this property is preserved by the RG evolution to any other scale.

To demonstrate this in a more explicit way, we study the renormalization of $\mathcal{L}_{\text {eff }}$. We assume that (3) includes all linearly independent operators which are allowed by global symmetries (Lorentz invariance, flavour quantum numbers etc.) and by their (canonical) mass dimension. Since Green's functions with single insertions of composite operators require only counterterms with at most the dimension of the inserted operator, $\mathcal{L}_{\text {eff }}$ can be renormalized multiplicatively by rewriting the effective Lagrangian in terms 
of renormalized operatorst

$$
O_{k}^{R}=Z_{k l} O_{l} .
$$

Operator mixing arises when $Z$ is not a diagonal matrix, and we say that an operator (or a set of operators) $O_{k}$ 'mixes into' $O_{l}$ if $Z_{k l} \neq 0$, i.e. if $O_{k}$ requires counterterms proportional to $O_{l}$.

Since $\mathcal{O}_{\mathrm{E}}$ mixes only into $\mathcal{O}_{\mathrm{E}}$, and since $\mathcal{O}_{\mathrm{B}}$ mixes only into $\mathcal{O}_{\mathrm{B}} \oplus \mathcal{O}_{\mathrm{E}}, Z$ and consequently the anomalous dimension matrix

$$
\gamma=Z \frac{d}{d \mu}\left(Z^{-1}\right)
$$

have block-triangular form, when written in a suitable basis of $\mathcal{O}_{\mathrm{E}} \oplus \mathcal{O}_{\mathrm{B}}$ and the remaining operators. Therefore, after the RG evolution [

$$
c_{i}\left(\mu_{2}\right)=\left[\exp \int_{g_{s}\left(\mu_{1}\right)}^{g_{s}\left(\mu_{2}\right)} \frac{\gamma^{T}(g)}{\beta(g)} d g\right]_{i j} c_{j}\left(\mu_{1}\right),
$$

the coefficients $c_{i}\left(\mu_{2}\right)$ of all $O_{i} \notin \mathcal{O}_{\mathrm{E}} \oplus \mathcal{O}_{\mathrm{B}}$ do indeed not depend on the initial values for the coefficients of the operators from $\mathcal{O}_{\mathrm{E}} \oplus \mathcal{O}_{\mathrm{B}}$.

Finally, we note the useful result by Joglekar and Lee [5] that gauge invariant operators mix only among themselves and into $\mathcal{O}_{\mathrm{E}} \oplus \mathcal{O}_{\mathrm{B}}$. Therefore, the operator basis for $\mathcal{L}_{\text {eff }}$ can be restricted to operators which are linearly independent of $\mathcal{O}_{\mathrm{E}} \oplus \mathcal{O}_{\mathrm{B}}$ and gauge-invariant. Of course, 'unphysical' operators which are not gauge-invariant and which are not contained in $\mathcal{O}_{\mathrm{E}} \oplus \mathcal{O}_{\mathrm{B}}$ can mix into physical operators. However, since the latter do not mix into these unphysical operators, their coefficients remain zero if the initial values vanish before the RG evolution. This is also illustrated by a recent RG calculation of Grinstein and Cho [23] where $O_{\mathrm{FP}}$ was included "for completeness": $O_{\mathrm{FP}}$ is not in $\mathcal{O}_{\mathrm{E}} \oplus \mathcal{O}_{\mathrm{B}}$ and indeed mixes into gauge-invariant operators.

\subsection{Equivalent effective Lagrangians at higher order}

When working beyond the linear approximation in the effective interactions one obviously has to include the effective interactions themselves in the adequate EOM (and one immediately wonders whether to use $\mathcal{L}_{\mathrm{o}}+\mathcal{L}_{\text {eff }}$ or $\mathcal{L}_{\mathrm{o}}+\mathcal{L}_{\text {eff }}-O_{\mathrm{EOM}}$ in the derivation). However, simply removing an operator which vanishes by the classical EOM, does not in general lead to a 'reduced' effective Lagrangian which is on-shell equivalent to $\mathcal{L}_{\text {eff }}$.

\footnotetext{
${ }^{4}$ Alternatively, the counterterms can be viewed as a renormalization of the couplings $c_{i}$; this approach is more convenient when working at higher order in $\mathcal{L}_{\text {eff }}$ (see appendix B).

${ }^{5}$ The exponential is $g$-ordered.

${ }^{6}$ Of course, one can always determine a 'reduced' effective Lagrangian $\mathcal{L}_{\text {red }}$, which is on-shell equivalent to $\mathcal{L}_{\text {eff }}$, by performing again the lengthy 'matching' procedure with on-shell amplitudes to fix all coefficients for a reduced (e.g. "canonical" [2]) set of operators.
} 
Green's functions with multiple insertions of $O_{\mathrm{EOM}}$ (and $\mathcal{L}_{\text {eff }}$ ) have 'non-trivial' contact

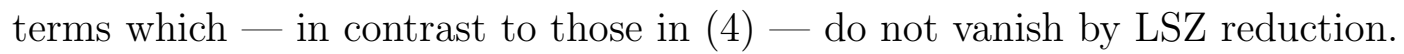

In practice, the effective couplings, collectively denoted by $g_{\text {eff }}$, are to be taken into account up to a certain order $M$ and the effective Lagrangian has the form $\mathcal{L}_{\text {eff }} \equiv$

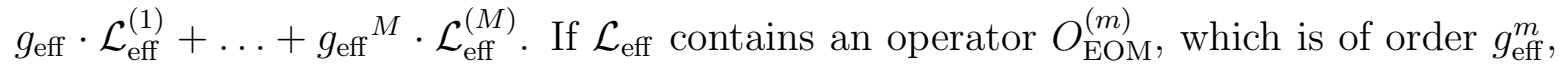
and which vanishes by the classical EOM derived from $\mathcal{L}_{\mathrm{o}}$, the 'reduction' of the effective Lagrangian by the EOM amounts essentially to the following task: Starting from $O_{\mathrm{EOM}}^{(m)}$, construct a 'reduced' effective Lagrangian,

$$
\mathcal{L}_{\text {red }}=\mathcal{L}_{\text {eff }}-O_{\mathrm{EOM}}^{(m)}+O\left(g_{\text {eff }}^{m+1}\right)
$$

which is on-shell equivalent to $\mathcal{L}_{\text {eff }}$ up to order $g_{\text {eff }}^{M}$.

In the linear case, $M=1$, and for $m=M$ one simply has to remove $O_{\mathrm{EOM}}^{(m)}$ from $\mathcal{L}_{\text {eff }}$. Otherwise, the first step in constructing $\mathcal{L}_{\text {red }}$ is to extend $O_{\mathrm{EOM}}^{(m)}$ recursively to an operator $O_{\text {EOM }}^{\prime}$ which vanishes by the EOM derived form $\mathcal{L}_{\mathrm{o}}+\mathcal{L}_{\text {eff }}^{\prime}$. Thereby, $\mathcal{L}_{\text {eff }}^{\prime}$ is defined iteratively by rewriting $\mathcal{L}_{\text {eff }}$ as

$$
\mathcal{L}_{\text {eff }}=\mathcal{L}_{\text {eff }}^{\prime}+O_{\mathrm{EOM}}^{\prime}
$$

The iteration starts with

$$
\begin{aligned}
\mathcal{L}_{\mathrm{eff}}^{\prime} & =\mathcal{L}_{\mathrm{eff}}-O_{\mathrm{EOM}}^{(m)}-O\left(g_{\mathrm{eff}}^{m+1}\right), \\
O_{\mathrm{EOM}}^{\prime} & =O_{\mathrm{EOM}}^{(m)}+O_{\mathrm{EOM}}^{(m+1)}+O\left(g_{\mathrm{eff}}^{m+2}\right),
\end{aligned}
$$

such that $O_{\mathrm{EOM}}^{(m)}+O_{\mathrm{EOM}}^{(m+1)}$ vanishes by the EOM from $\mathcal{L}_{\mathrm{o}}+\mathcal{L}_{\text {eff }}^{\prime}\left(\right.$ keeping in $\mathcal{L}_{\text {eff }}^{\prime}$ only terms of order $g_{\text {eff }}$ ).

We may assume that $O_{\mathrm{EOM}}^{(m)}$ contains inverse propagators only through powers of $\delta_{\mathrm{E}} \mathcal{L}_{\mathrm{o}}$, where $\delta_{\mathrm{E}} \equiv \delta / \delta \Phi-\partial_{\mu} \delta / \delta\left(\partial_{\mu} \Phi\right)$. Then, $O_{\mathrm{EOM}}^{\prime}$ can be chosen to contain inverse propagators only through powers of $\delta_{\mathrm{E}}\left(\mathcal{L}_{\mathrm{o}}+\mathcal{L}_{\text {eff }}^{\prime}\right)$. For instance, for $O_{\mathrm{EOM}}^{(m)}=Q \cdot\left(\delta_{\mathrm{E}} \mathcal{L}_{\mathrm{o}}\right)^{2}$ one would set $O_{\mathrm{EOM}}^{(m+1)}=2 Q \cdot \delta_{\mathrm{E}} \mathcal{L}_{\mathrm{o}} \cdot \delta_{\mathrm{E}} \mathcal{L}_{\text {eff }}^{(1)}$, etc.

Before removing $O_{\mathrm{EOM}}^{\prime}$ we have to take into account its on-shell contributions through non-trivial contact terms on the r.h.s. of (2) [or its iteration in the case that $O_{\mathrm{EOM}}^{\prime}$ contains higher powers of $\left.\delta_{\mathrm{E}}\left(\mathcal{L}_{\mathrm{o}}+\mathcal{L}_{\text {eff }}^{\prime}\right)\right]$. The amplitudes are evaluated from the first $M$ terms of the Gell-Mann-Low series

$$
\begin{aligned}
\left\langle T \exp \left\{i \int \mathcal{L}_{\mathrm{eff}}(y) d y\right\} X\left(x_{1}, \ldots\right)\right\rangle & =\sum_{n=1}^{M} \frac{i^{n}}{n !} \int d y_{1} \ldots d y_{n}\left\langle T \mathcal{L}_{\mathrm{eff}}\left(y_{1}\right) \ldots \mathcal{L}_{\mathrm{eff}}\left(y_{n}\right) X\left(x_{1}, \ldots\right)\right\rangle \\
& +O\left(g_{\mathrm{eff}}^{M+1}\right)
\end{aligned}
$$

and all on-shell contributions of $O_{\mathrm{EOM}}^{\prime}$ remaining on the total r.h.s. of (17) correspond to (sub-)diagrams with the following properties: (a) All internal lines are canceled by 
inverse propagators in the $O_{\mathrm{EOM}}^{\prime}$ and there is at least one internal line, (b) none of the inverse propagators in the $O_{\mathrm{EOM}}^{\prime}$ acts on an external line from $X\left(x_{1}, \ldots\right)$, and (c) all vertices from $\mathcal{L}_{\mathrm{o}}+\mathcal{L}_{\text {eff }}^{\prime}$ have at least two lines, which are canceled by inverse propagators from $O_{\mathrm{EOM}}^{\prime}$.

Obviously, one can just choose suitable subdiagrams with property (a). If (b) were not true, the diagram would not survive LSZ reduction; and (c) is a consequence of (a) and the assumption that $O_{\mathrm{EOM}}^{\prime}$ contains inverse propagators only through powers of $\delta_{\mathrm{E}}\left(\mathcal{L}_{\mathrm{o}}+\mathcal{L}_{\text {eff }}^{\prime}\right)$.

Sub-diagrams with property (a) correspond to local vertices which are obtained by shrinking the internal lines to a point. In this way one can represent the contribution of each contact term by a local operator, and we denote the sum of all these 'contact operators' by $C\left[O_{\mathrm{EOM}}^{\prime}\right]$. More precisely, $C\left[O_{\mathrm{EOM}}^{\prime}\right]=C_{2}+C_{3}+\cdots$, where the $C_{n}$ are determined by

$$
\begin{aligned}
& i\left\langle T C_{n}\left(y_{1}\right) X\left(x_{1}, \ldots\right)\right\rangle \prod_{\nu=2}^{n} i \delta\left(y_{1}-y_{n}\right) \stackrel{!}{=} \\
& \left.\quad \frac{i^{n}}{n !}\left\langle T O_{\mathrm{EOM}}^{\prime}\left(y_{1}\right) \ldots O_{\mathrm{EOM}}^{\prime}\left(y_{n}\right) \exp \left\{i \int \mathcal{L}_{\text {eff }}^{\prime}(y) d y\right\} X\left(x_{1}, \ldots\right)\right\rangle\right|_{(a)-(c)}
\end{aligned}
$$

for arbitrary elementary fields, $X\left(x_{1}, \ldots\right)$, but with the r.h.s. restricted to the contributions of maximal connected diagrams satisfying (a)-(c). Working up to order $g_{\mathrm{eff}}^{M}$, only a finite number of contact operators appears in $C\left[O_{\mathrm{EOM}}^{\prime}\right]$.

Finally, the 'reduced' effective Lagrangian is obtained by replacing $O_{\mathrm{EO}}^{\prime}$ by its contact operators

$$
\mathcal{L}_{\text {red }} \equiv \mathcal{L}_{\text {eff }}^{\prime}+C\left[O_{\text {EOM }}^{\prime}\right]
$$

Note that $C\left[O_{\mathrm{EOM}}^{\prime}\right]$ depends in a non-linear way on $O_{\mathrm{EOM}}^{\prime}$ (or its coefficient, which we have absorbed in $\left.O_{\mathrm{EOM}}^{\prime}\right)$. If $\mathcal{L}_{\text {eff }}=\mathcal{L}_{\text {eff }}^{\prime}+\lambda O_{\mathrm{EOM}}^{\prime}$, then $\mathcal{L}_{\text {red }}=\mathcal{L}_{\text {eff }}^{\prime}+C\left[\lambda O_{\mathrm{EOM}}^{\prime}\right]$, which is given in terms of the contact operators from (18) by $C\left[\lambda O_{\mathrm{EOM}}^{\prime}\right]=\lambda^{2} C_{2}+\lambda^{3} C_{3}+\cdots$.

To illustrate the procedure, we consider a simple example: $\mathcal{L}_{\mathrm{o}}=-\frac{1}{2} \Phi\left(\partial^{2}+m^{2}\right) \Phi$ $+\bar{\Psi} \not \partial \Psi$ describes a massive scalar and a massless fermion, and $\mathcal{L}_{\text {eff }}=g_{\text {eff }} \Psi \Psi \partial^{2} \Phi$ are their (effective) interactions. On-shell amplitudes at order $g_{\text {eff }}$ (e.g. one scalar decaying into two fermions) are obviously recovered by $\mathcal{L}_{\text {red }}^{(1)}=-g_{\text {eff }} \bar{\Psi} \Psi m^{2} \Phi=\mathcal{L}_{\text {eff }}-O_{\mathrm{EOM}}^{(1)}$, where $O_{\mathrm{EOM}}^{(1)} \equiv g_{\text {eff }} \bar{\Psi} \Psi\left(\partial^{2}+m^{2}\right) \Phi$. At second order in $g_{\text {eff }}, \mathcal{L}_{\text {eff }}$ and $\mathcal{L}_{\text {red }}^{(1)}$ are not onshell equivalent. In particular, $\mathcal{L}_{\mathrm{o}}+\mathcal{L}_{\text {red }}^{(1)}$ does not yield the correct amplitude for the two-fermion scattering, $A=-i g_{\text {eff }}^{2} \frac{s^{2}}{s-m^{2}}+(s \leftrightarrow t)$, where $s$ and $t$ are the Mandelstam variables. Following the above procedure, we set

$$
O_{\mathrm{EOM}}^{\prime}=\mathcal{O}_{\mathrm{EOM}}^{(1)}+\frac{\delta \mathcal{L}_{\text {eff }}}{\delta \Phi}=g_{\mathrm{eff}} \bar{\Psi} \Psi\left(\partial^{2}+m^{2}\right) \Phi+g_{\mathrm{eff}}^{2} \bar{\Psi} \Psi \bar{\Psi} \Psi m^{2},
$$

and from (18), we find $C\left[O_{\mathrm{EOM}}^{\prime}\right]=g_{\mathrm{eff}}^{2} / 2 \bar{\Psi} \Psi\left(\partial^{2}+m^{2}\right)(\bar{\Psi} \Psi)$. One readily verifies that $\mathcal{L}_{\text {red }} \equiv \mathcal{L}_{\text {eff }}-O_{\text {EOM }}^{\prime}+C\left[O_{\text {EOM }}^{\prime}\right]$ indeed yields the same physical amplitudes as $\mathcal{L}_{\text {eff }}$. In 
the particular example at hand, this is the case even at arbitrary order in $g_{\text {eff }}$, because

$O_{\mathrm{EOM}}^{\prime}$ vanishes identically by the EOM derived from $\mathcal{L}_{\mathrm{o}}+\mathcal{L}_{\text {eff }}-O_{\mathrm{EOM}}^{\prime}$ and there are no further contact terms at higher orders.

When working at the loop level, the above reduction procedure remains essentially the same: All expressions are to be understood as referring to the basic part of the renormalized (effective) Lagrangian, and $C\left[O_{\mathrm{EOM}}^{\prime}\right]$ is still given by the tree result because loop diagrams with property (a) vanish in dimensional regularization. Since renormalized Green's functions of $O_{\mathrm{EOM}}^{\prime}$ obey the same relations (derived from the basic part of the Lagrangian) as the bare ones, $\mathcal{L}_{\text {red }}^{R}$ is again on-shell equivalent to $\mathcal{L}_{\text {eff }}^{R}$; and the $\mathrm{RG}$ evolution preserves this equivalence. In contrast to the linear approximation in $g_{\text {eff }}$, it would be hard to demonstrate this in an explicit way because the reduction by the EOM, and the RG evolution (see, for instance, ref. [6]) are non-linear operations on $\mathcal{L}_{\text {eff }}$.

For the calculation of amplitudes at higher order in $g_{\text {eff }}$, the Green's functions with multiple insertions of $\mathcal{L}_{\text {eff }}$ are actually needed only after integration over the positions of the effective operators [see (17)] and not in their general form. Therefore, it is sufficient and in fact more convenient to view the counterterms for $\mathcal{L}_{\text {eff }}$ as a renormalization of the effective couplings (see appendix B for more details), rather than of the operators as in $(13)$.

\section{Effective treatment of rare B-decays}

We apply now the results of the previous section to rare B-decays; in particular, we consider hadronic (and in less detail also radiative) $b \rightarrow s$ transitions. (The analogous $b \rightarrow d$ modes follow simply from replacing $V_{t s}$ by $V_{t d}$.) Since the momenta and masses of the external particles are at most of the order of the B-mass (and therefore much smaller than the mass scales governing the propagation of virtual $t$-quarks or $W$-bosons), it is possible to treat these processes in the framework of an effective low energy theory [1]. The phenomenological procedure in this framework consists of three steps: The derivation of the adequate effective Lagrangian, the RG evolution and finally, the evaluation of the hadronic matrix elements of the effective operators.

In step one, the heavy particles are integrated out. The resulting effective action contains only the fields of the light particles, however, possibly in a non-local way. In order to obtain a local effective Lagrangian one has to perform an operator product expansion [24] in the effective action. If strong (and higher order electroweak) interactions are absent, this simply corresponds to a Taylor expansion in the external momenta. Otherwise, the coefficient functions of the local operators in the effective Lagrangian are determined by a 'matching' condition: Appropriate amplitudes calculated in the effec- 
tive theory are equated with those of the full theory. As soon as divergent loop diagrams of effective operators are involved a renormalization scheme has to be specified. The coefficients will then depend on this scheme and, in particular, on the renormalization scale $\mu$. A natural choice for $\mu$ to perform the 'matching' is of the order of the heavy masses, $M_{W}$ in our case. A scale of this size allows to take into account (short-distance) QCD effects perturbatively and minimizes logarithmic corrections from higher order loops with heavy particles.

On the other hand, the matrix elements of the effective operators involve logarithms of the ratio of $\mu$ to the typical mass scale of the low-energy process, which is of order $m_{b}$ in our case. These large logarithms certainly distort a simple perturbative treatment of the matrix elements and are removed by the RG evolution in step two: The physical contents of the effective Lagrangian, 'matched' at $\mu \approx M_{W}$, is translated to an effective theory with a renormalization scale $\mu \approx m_{b}$. Thereby, the RG evolution of the coefficients [see (15)] allows to improve the (perturbative) treatment of short-distance QCD corrections by summing up all powers of $\alpha_{s} \ell n \frac{\mu^{2}}{M_{W}^{2}}$.

The RG evolution is straightforward in principle, however, the explicit calculation is rather tedious and involves subtle technical details. There are numerous important contributions (see, for instance, ref. [25] and [17-20]) and we refer to Buras et al. 20] and Misiak [26] for the most recent and complete results. Here, we rather discuss some details of the derivation and 'matching' of the effective Lagrangian. This provides the starting point for further phenomenological studies that intend to include the RG improvement.

The third step, the evaluation of the matrix elements of the effective operators with realistic hadron states is, of course, the most difficult task because it requires genuine non-perturbative methods (or drastically simplifying assumptions about the quark and gluon contents of the hadrons together with some model to describe their binding effects). We shall completely ignore non-perturbative effects and stay on the parton level throughout.

\subsection{Effective vertices from penguin loops}

In this subsection, we describe one contribution to the effective Lagrangian: Effective vertices resulting from one-particle-irreducible (1PI) loop diagrams with internal top quark. The application of the EOM already at this stage drastically simplifies further calculations (e.g. of the 'full' amplitudes required for the matching). The 'matching' procedure to properly define the complete effective Lagrangian is postponed to the next subsection; only then, the effective operators corresponding to $W$-exchange diagrams involving only light quarks, like $b \rightarrow s u \bar{u}$ and $s c \bar{c}$, will be introduced (and renormalized). 
At leading order in $1 / M_{W}^{2}$ the loop diagrams to be considered are 'penguins' with up to three gluons emitted from the $t$-line inside the loop (diagrams with photons and leptons will be discussed below). They are renormalized within the full theory by counterterms in the original electroweak Lagrangian (which in fact cancel by the GIM mechanism). As long as the penguins do not appear as subdiagrams in two- (or more-) loop amplitudes, the external momenta are bound by $m_{b}\left(\ll m_{t}\right.$ or $\left.M_{W}\right)$; and we can neglect any momentum dependence which is higher order in $m_{b}^{2} /\left(m_{t}^{2}\right.$ or $\left.M_{W}^{2}\right)$. Then, the 1PI diagrams are equivalent to (local) effective vertices and can be summarized in terms of a 'penguin' Lagrangian

$$
\mathcal{L}_{\mathrm{P}}=-4 \frac{G_{F}}{\sqrt{2}} V_{t b} V_{t s}^{*} \sum_{i} c_{i}^{\prime} P_{i} .
$$

We choose the following set of gauge-invariant operators of dimension up to six (see appendix $\mathrm{C}$ for additional details)

$$
\begin{aligned}
P_{1} & =\frac{g_{s}}{16 \pi^{2}} \cdot \bar{s} \gamma_{\nu} L \frac{\lambda^{a}}{2} b \cdot\left(D_{\mu} G^{\mu \nu}\right)_{a}, \\
P_{2} & =\frac{g_{s}}{16 \pi^{2}} \cdot \bar{s} \sigma_{\mu \nu}\left(m_{b} R+m_{s} L\right) \frac{\lambda^{a}}{2} b G_{a}^{\mu \nu} \quad\left(\equiv O_{g}\right) \\
P_{3} & =\frac{g_{s}}{16 \pi^{2}} \cdot \bar{s}\left\{i \not D, \sigma_{\mu \nu} G_{a}^{\mu \nu} \frac{\lambda^{a}}{2}\right\} L b \\
P_{4} & =\frac{g_{s}}{16 \pi^{2}} \cdot \bar{s}\left[i \not D, \sigma_{\mu \nu} G_{a}^{\mu \nu} \frac{\lambda^{a}}{2}\right] L b \\
P_{5} & =\frac{1}{16 \pi^{2}} \cdot \bar{s} i \not D \not D \not D L b \\
P_{6} & =\frac{1}{16 \pi^{2}} \cdot \bar{s} \not D \not D\left(m_{b} R+m_{s} L\right) b \\
P_{7(L, R)} & =\frac{1}{16 \pi^{2}} \cdot \bar{s} i \not D M_{W}^{2}(L, R) b \\
P_{8(L, R)} & =\frac{1}{16 \pi^{2}} \cdot \bar{s} M_{W}^{2}\left(m_{s} L, m_{b} R\right) b
\end{aligned}
$$

where $b$ and $\bar{s}$ denote the quark fields, and the covariant derivatives are defined in (5) and (10).

The coefficients $c_{i}^{\prime}$ are functions of the variable $x=\frac{m_{t}^{2}}{M_{W}^{2}}$ (see appendix $\mathrm{C}$ ), and $c_{7 L, R}^{\prime}$ and $c_{8 L, R}^{\prime}$ depend also on the renormalization scheme of the full theory. Since the $b$ - and $s$-momenta enter $P_{4}$ in an antisymmetric way, $c_{4}^{\prime}$ is suppressed by an additional factor of the order $m_{b}^{2} / M_{W}^{2}$ and can be neglected. We also note that only $c_{1}^{\prime}$ has a logarithmic term, $c_{1}^{\prime} \sim-\frac{2}{3} \ln x+O(x)$, which would dominate for $x \longrightarrow 0$. All other coefficients approach a constant value in this (unrealistic) limit.

All coefficient functions $c_{i}^{\prime}$ are uniquely determined by the (off-shell) digrams for the $\bar{s} b g$ vertex and the $\bar{s} b$ self energy; a calculation of the 1 PI diagrams for $b \rightarrow s g g$ and $b \rightarrow s g g g$ is not necessary. In turn, $\mathcal{L}_{\mathrm{P}}$ provides the $1 \mathrm{PI}$ vertices for all $b \rightarrow s$ transitions involving up to three gluons (effective operators for more than three gluons have dimension higher than six and are suppressed by powers of $1 / M_{W}^{2}$ or $1 / m_{t}^{2}$ ). This is simply a consequence of gauge invariance which is incorporated in $\mathcal{L}_{\mathrm{P}}$ by using manifestly gauge invariant operators. In momentum space the corresponding relations among the 
various effective vertices are much less transparent and the first complete treatments of $b \rightarrow s g g$ [11] exploited lengthy Slavnov-Taylor identities or a generalization of Low's low energy theorem.

When $\mathcal{L}_{\mathrm{P}}$ enters only at first order, we can take advantage of the EOM (5) and (10), and considerably reduce $\mathcal{L}_{\mathrm{P}}: P_{5}, P_{6}$ and $P_{7 L, R}$ become proportional to the flavour off-diagonal mass terms $P_{8 L, R}$ with coefficients that cancel when the full theory is renormalized on shell[. Applying the EOM to $P_{1}$, one obtains the four-quark operator

$$
O_{P}=\bar{s} \gamma_{\nu} L \frac{\lambda^{a}}{2} b \sum_{q u a r k s} \bar{q} \gamma^{\nu} \frac{\lambda^{a}}{2} q
$$

(plus additional operators from gauge-fixing and ghosts, which may be dropped according to the discussion of section 2). Finally, $P_{3}$ and $P_{4}$ yield color-magnetic dipole operators equal to $P_{2}\left(P_{4}\right.$ yields actually a different chiral structure if $m_{s}$ is not neglected).

After this reduction with the help of the EOM, $P_{2} \equiv O_{g}$ is the only operator which contains a gluon field; and $O_{P}$ and $O_{g}$ are the only two operators remaining in $\mathcal{L}_{\mathrm{P}}$. Their coefficients

$$
\begin{aligned}
c_{P}^{\prime} & =-\frac{g_{s}^{2}}{16 \pi^{2}} c_{1}^{\prime}=-\frac{g_{s}^{2}}{16 \pi^{2}}\left(F_{1}(x)+\frac{1}{9}\right), \\
c_{g}^{\prime} & =c_{2}^{\prime}+c_{3}^{\prime}=-\frac{1}{2}\left(F_{2}(x)-\frac{2}{3}\right)
\end{aligned}
$$

can be expressed in terms of Inami-Lim functions [27] from the $\bar{s} b g$-vertex $\left[F_{1}\right.$ and $F_{2}$, as defined in appendix $\mathrm{D}$, have no constant terms for $x \rightarrow 0$; hence the explicit constants in (22), which is irrelevant in all physical applications because of the GIM mechanism].

Radiative decays require additional penguin diagrams with one, two or three photons coupling to the $W$ and to the unphysical Higgs inside the loop. (By choosing a nonlinear gauge for the electroweak sector on can avoid diagrams with a photon- $W$-Higgs coupling. Otherwise, these give rise to additional operators [11] which either vanish on shell, or have coefficients that are independent of the internal quark mass and, therefore, cancel by to the GIM mechanism.) The resulting effective vertices correspond to four new operators, $\tilde{P}_{1} \ldots \tilde{P}_{4}$, which are obtained from $P_{1} \ldots P_{4}$ by the replacements $G_{\mu \nu}^{a} \rightarrow F_{\mu \nu}$, $\frac{\lambda^{a}}{2} \rightarrow 1$ and $g_{s} \rightarrow e$. Among their coefficients, only $\tilde{c}_{1}^{\prime}$ has a logarithmic behavior, $\tilde{c}_{1}^{\prime} \longrightarrow-\frac{4}{9} \ln x$, while all others become constant for $x \longrightarrow 0$. By applying the EOM to $\tilde{P}_{1} \ldots \tilde{P}_{4}$ (and to $P_{3} \ldots P_{7(L, R)}$, whose covariant derivatives include, of course, also the photon field) all local vertices for $b \rightarrow g \gamma$ or $b \rightarrow s \gamma \gamma$ are removed; the only operator

7 The on-shell renormalization conditions are equivalent to the requirement that all flavour offdiagonal mass terms vanish already when the EOM are applied either only to $\bar{s}$ or only to $b$. 
containing a photon field is $O_{\gamma} \equiv \tilde{P}_{2}$ and its coefficient is an other well-known Inami-Lim function

$$
c_{\gamma}^{\prime}=\tilde{c}_{2}^{\prime}+\tilde{c}_{3}^{\prime}=-\frac{1}{2}\left(\tilde{F}_{2}(x)-\frac{23}{18}\right) .
$$

At order $\alpha \cdot G_{F}$, additional penguin diagrams with $Z$-bosons (decaying into leptons), and $W$-box-diagrams arise. The resulting effective four-Fermi vertices couple $\bar{s} b$ to neutrinos, and to the vector and axial current of the charged leptons. $c_{\gamma}^{\prime}$ and the coefficients of these semi-leptonic operators depend on the gauge that is chosen in the electroweak sector. Of course, all (four-Fermi) operators that remain after applying the EOM to $\tilde{P}_{1}$, have gauge-independent coefficients (see also refs. 27, 28]).

$\mathcal{L}_{\mathrm{P}}$ turns out to be useful even for the treatment of diagrams with light internal quarks. Neglecting terms of order $\frac{q^{2}}{M_{W}^{2}} \ll 1$, where $q^{2}$ collectively denotes the external momenta, one can always split the amplitude for a penguin diagram with an internal quark $i=u, c, t$ into two pieces:

$$
A^{(i)}=\sum_{k} c_{k}^{\prime}\left(\frac{m_{i}^{2}}{M_{W}^{2}}\right) \cdot\left\langle f\left|P_{k}\right| b\right\rangle^{\text {tree }}+\Delta \mathrm{A}\left(\frac{q^{2}}{m_{i}^{2}}\right) .
$$

For the heavy internal top quark, only the first term, arising from the effective vertices in $\mathcal{L}_{\mathrm{P}}$, is relevant. $\Delta \mathrm{A}$ vanishes with powers of $q^{2} / m_{i}^{2}$ for momenta small compared to the internal quark mass. For light internal quarks the coefficients in the first (local) term are evaluated at the appropriate value of $m_{i}^{2}$, while the remaining momentum dependence of the amplitude is contained in $\Delta \mathrm{A}$ (including, for instance, threshold singularities in $\left.q^{2} / m_{i}^{2}\right)$.

$\Delta \mathrm{A}$ being the difference of the amplitudes at different external momenta is worked out most conveniently by using the four-Fermi approximation instead of the full $W$ propagator; no UV divergence arises in $\Delta \mathrm{A}$ and no renormalization is necessary. For $b \rightarrow s q \bar{q}$ 早

$$
\Delta \mathrm{A}\left(\frac{q^{2}}{m_{i}^{2}}\right)=\Delta F_{1}\left(\frac{q^{2}}{m_{i}^{2}}\right) \cdot\left\langle s q \bar{q}\left|O_{P}\right| b\right\rangle^{\text {tree }}
$$

differs from the local part just by a momentum dependent form factor $\Delta F_{1}$ (see appendix D). In general, $\Delta \mathrm{A}$ consists of matrix elements of local operators, some of which may have dimensions higher than six, multiplied by momentum dependent form factors.

The decomposition (24) is very convenient for processes like $b \rightarrow$ sgg etc., where the momentum dependence $\Delta \mathrm{A}$ contributes significantly in large regions of phase space and, hence, can not be neglected. In the effective theory the momentum dependent part $\Delta \mathrm{A}$ is recovered by loop-level matrix elements of effective four-Fermi operators.

\footnotetext{
${ }^{8}$ Eqs. (24) and (25) should be contrasted to the more complicated result of ref. 29, which keeps terms of order $q^{2} / M_{W}^{2}$, but is not applicable for large $m_{i} \gtrsim M_{W}$. In (24) the Inami-Lim function from $c_{P}^{\prime}$ gives always the correct mass dependence.
} 
However, due to their renormalization, the correspondence is not straightforward and has to be clarified in order to incorporate correction factors from short-distance QCD in a systematic way. To this end, we take a closer look at the 'matching' conditions that define the coefficients of the operators in the effective Lagrangian.

\subsection{Matching of the effective Lagrangian}

In addition to the vertices from $\mathcal{L}_{\mathrm{P}}$ we consider now the effective operators for the usual $W$-exchange. Writing the effective Lagrangian as

$$
\mathcal{L}_{\text {eff }}=4 \frac{G_{F}}{\sqrt{2}}\left(V_{c b} V_{c s}^{*} \mathcal{L}_{c}+V_{u b} V_{u s}^{*} \mathcal{L}_{u}\right)
$$

we need to discuss only $\mathcal{L}_{c}=\sum_{k} c_{k} O_{k}^{R}$ in the following; $\mathcal{L}_{u}$ is simply obtained from $\mathcal{L}_{c}$ by replacing the charm field (and mass) everywhere in $O_{k}$ (and $c_{k}$ ). In fact, since $V_{u b} V_{u s}^{*} \ll$ $V_{c b} V_{c s}^{*}$, one may neglect $\mathcal{L}_{u}$ in most phenomenological applications not concerned with $\mathrm{CP}$ violation. Note that all terms proportional to $V_{t b} V_{t s}^{*}$ (from internal $t$-quarks) are distributed to $\mathcal{L}_{c}$ and $\mathcal{L}_{u}$ via the unitarity of the CKM matrix.

The local four-Fermi limit of the usual tree-level $W$-exchange, $O_{2}=\bar{s}_{\alpha} \gamma^{\mu} L c_{\alpha}$. $\bar{c}_{\beta} \gamma_{\mu} L b_{\beta}$, mixes with the operator $O_{1}=\bar{s}_{\alpha} \gamma^{\mu} L c_{\beta} \cdot \bar{c}_{\beta} \gamma_{\mu} L b_{\alpha}$ with reversed color structure. Both mix into further four-quark operators, $O_{3} \ldots O_{6}$, which carry various color and chiral structures 9 , and are related to the penguin operator $O_{P}=\frac{1}{2}\left[O_{4}+O_{6}-\frac{1}{N}\left(O_{3}+O_{5}\right)\right]$. At the two-loop level the four-quark operators mix into the color-magnetic moment operator $O_{g}$ [19, 26].

According to the discussion of section 2, all operators which vanish by the EOM or can be written as BRS variations are irrelevant in the present physical application (first order in $\mathcal{L}_{\text {eff }}$ !). In this sense, $O_{1} \ldots O_{6}$ and $O_{g}$ form a complete set of the dimension-five or -six operators for hadronic $b \rightarrow s$ transitions. In addition, $O_{\gamma}$, and the semi-leptonic four-Fermi operators, and electromagnetic analogs of $O_{3} \ldots O_{6}$ have to be included in order to describe radiative and semi-leptonic decays, or to take into account mixing at order $\alpha_{\mathrm{QED}}$.

To obtain a systematic treatment of QCD corrections, the (yet unknown) coefficients at $\mu=M_{W}$ are expanded in powers of $g_{s}^{2}$

$$
c_{i}\left(M_{W}\right) \equiv c_{i}^{(0)}+g_{s}^{2} c_{i}^{(1)}+\cdots .
$$

Electromagnetic couplings are included by rewriting $e=g_{s} \cdot \frac{e}{g_{s}}$ and treating $\frac{e}{g_{s}}$ as an independent (running) parameter.

${ }^{9}$ We are here not interested in the explicit expressions for these operators; they can be found in e.g. [20]. Note, however, that the Fierz ordering of the operators should be kept fixed throughout all calculations in $d \neq 4$ dimensions. 
If the operators are defined with appropriate factors of $1 / g_{s}^{2}$ [26] they do not mix at zeroth order and the expansion of the anomalous dimension matrix starts at order $g_{s}^{2}$

$$
\gamma_{k l} \equiv g_{s}^{2} \cdot \gamma_{k l}^{(1)}+g_{s}^{4} \cdot \gamma_{k l}^{(2)}+\cdots
$$

The RG evolution (15) can be written as

$$
c_{i}(\mu)=\left(E_{i j}^{(L L)}(\mu)+g_{s}^{2} \cdot E_{i j}^{(N L L)}(\mu)+\cdots\right) c_{j}\left(M_{W}\right),
$$

where the evolution matrices $E$ sum up all orders of the product $\alpha_{s} \cdot \log \left(M_{W}^{2} / \mu^{2}\right)$. Since the logarithm may be large, this product is not expanded in $g_{s}$, but rather treated as an independent parameter.

In leading- $\log (\mathrm{LL})$ approximation all $O\left(g_{s}^{2}\right)$ corrections are neglected, and the $c_{i}^{(0)}\left(M_{W}\right)$ are determined by the matching condition

$$
A^{\text {full }}(b \rightarrow f) \stackrel{!}{=} c_{i}^{(0)}\left(M_{W}\right) \cdot\left\langle f\left|O_{i}^{R}\right| b\right\rangle^{(0)}+O\left(g_{s}^{2}\right) .
$$

were the amplitude in the full theory, $A^{\text {full }}$, and the matrix elements, $\left\langle f\left|O_{i}^{R}\right| b\right\rangle^{(0)}$, have to be evaluated to order $g_{s}^{0}$ (or to order $g_{s}$ if $A^{\text {full }}=O\left(g_{s}\right)$, like for $b \rightarrow s g$ ).

Using appropriate final states, (27) yields

$$
\begin{aligned}
& c_{2}^{(0)}=-1 \\
& c_{g}^{(0)}=c_{g}^{\prime}\left(\frac{m_{t}^{2}}{M_{W}^{2}}\right)-c_{g}^{\prime}\left(\frac{m_{c}^{2}}{M_{W}^{2}}\right)=-\frac{1}{2} F_{2}\left(\frac{m_{t}^{2}}{M_{W}^{2}}\right)+O\left(\frac{m_{c}^{2}}{M_{W}^{2}}\right), \\
& c_{\gamma}^{(0)}=c_{\gamma}^{\prime}\left(\frac{m_{t}^{2}}{M_{W}^{2}}\right)-c_{\gamma}^{\prime}\left(\frac{m_{c}^{2}}{M_{W}^{2}}\right)=-\frac{1}{2} \tilde{F}_{2}\left(\frac{m_{t}^{2}}{M_{W}^{2}}\right)+O\left(\frac{m_{c}^{2}}{M_{W}^{2}}\right),
\end{aligned}
$$

while all other coefficients are zero at $\mu=M_{W}$.

Penguin contributions are order $\alpha_{s}$ in the full theory, and do in LL approximation not enter the effective theory at $\mu=M_{W}$. However, when evolving to $\mu<M_{W}, O_{2}$ mixes into $O_{3} \ldots O_{6}\left(\gamma_{23}^{(1)}=-\frac{1}{2 N} \frac{2}{3} \frac{1}{8 \pi^{2}}\right.$ etc. $)$, and if one expands their coefficients to leading order in $\alpha_{s}$, they combine to $O_{P}$ with the coefficient

$$
c_{P}(\mu)=\frac{\alpha_{s}(\mu)}{4 \pi} \cdot \frac{2}{3} \ln \left(\frac{\mu^{2}}{M_{W}^{2}}\right) \cdot c_{2}^{(0)}\left(M_{W}\right)+\alpha_{s} \cdot O(1)+O\left(\alpha_{s}^{2}\right) .
$$

For three-body decays like $b \rightarrow$ sgg etc., and for CP-violating asymmetries in charged B-decays, one-loop matrix elements of the effective operators are of basic interest because they give rise to significant momentum dependent contributions [11] and to the crucial absorptive phase of the amplitudes [10, 12, 30]. As an example, we consider the matrix element

$$
\left\langle s d \bar{d}\left|O_{2}^{R}\right| b\right\rangle^{(1)}=-\frac{\alpha_{s}(\mu)}{4 \pi}\left[\Delta F_{1}\left(\frac{q^{2}}{m_{c}^{2}}\right)-\frac{2}{3}\left(\xi_{\mathrm{R}}+\ln \frac{m_{c}^{2}}{\mu^{2}}\right)\right] \cdot\left\langle s d \bar{d}\left|O_{P}\right| b\right\rangle^{(0)},
$$


which corresponds to a penguin diagram with an internal $c$-quark in the full theory. Here, $q^{2}$ is the invariant mass of the $d \bar{d}$-pair and $\xi_{\mathrm{R}}=1$ for naive dimensional regularization with $\overline{m s}$ subtraction. The one-loop matrix element (30) differs from the pure momentum dependence $\Delta F_{1}$ of the full amplitude [see (25)] by additional terms, which originate from the renormalization. Eqs. (29) and (30) demonstrate how the RG evolution from $\mu=M_{W}$ to $\mu \approx m_{b}$ moves the large logarithms from the matrix elements into the coefficient functions.

If the effective theory is matched only with $O\left(g_{s}\right)$ precision and if the RG evolution is performed only in LL approximation, one can not expect that higher order effects, like the $O\left(\alpha_{s}\right)$ matrix element in (30), exactly reproduce the full theory. The appearance of $\xi_{\mathrm{R}}$ clearly indicates some arbitrariness, because it depends on the details of the renormalization scheme. On the other hand, the logarithmic mass dependence and the proper momentum dependence is recovered already at this stage: Combining (29) and (30) yields for $b \rightarrow s d \bar{d}$

$$
A^{\mathrm{eff}} \sim \frac{\alpha_{s}}{4 \pi}\left[O(1)-\frac{2}{3} \ell n \frac{m_{c}^{2}}{M_{W}^{2}}+\Delta F_{1}\left(\frac{q^{2}}{m_{c}^{2}}\right)\right] \cdot\left\langle s d \bar{d}\left|O_{P}\right| b\right\rangle^{(0)}+c_{g}^{(0)} \cdot\left\langle s d \bar{d}\left|O_{g}\right| b\right\rangle,
$$

which is to be compared with the full theory [see (24)]

$$
A^{\text {full }} \sim \frac{\alpha_{s}}{4 \pi}\left[F_{1}\left(\frac{m_{c}^{2}}{M_{W}^{2}}\right)-F_{1}\left(\frac{m_{t}^{2}}{M_{W}^{2}}\right)+\Delta F_{1}\left(\frac{q^{2}}{m_{c}^{2}}\right)\right] \cdot\left\langle s d \bar{d}\left|O_{P}\right| b\right\rangle^{(0)}+c_{g}^{(0)} \cdot\left\langle s d \bar{d}\left|O_{g}\right| b\right\rangle .
$$

Recalling $F_{1}(x)=-\frac{2}{3} \ell n x+O(x)$, the two expressions agreetण within "logarithmic precision"; they differ only by non-logarithmic terms of order $\alpha_{s} \cdot 1$ and, of course, exhibit the same momentum dependence.

To go beyond this precision, one has to include the $O\left(g_{s}^{2}\right)$ corrections in the initial values of the coefficients and the RG evolution has to be performed in next-to-leading$\log (\mathrm{NLL})$ approximation. To this end, the $\gamma_{k l}^{(2)}$ are needed to evaluate the evolution matrix $E^{(N L L)}$, and the $c_{i}^{(1)}\left(M_{W}\right)$ are obtained by solving

$$
A^{\text {full }}(b \rightarrow f) \stackrel{!}{=} c_{i}^{(0)} \cdot\left(\left\langle f\left|O_{i}^{R}\right| b\right\rangle^{(0)}+g_{s}^{2} \cdot\left\langle f\left|O_{i}^{R}\right| b\right\rangle^{(1)}\right)+g_{s}^{2} c_{i}^{(1)} \cdot\left\langle f\left|O_{i}^{R}\right| b\right\rangle^{(0)}+O\left(g_{s}^{4}\right),
$$

where $A^{\text {full }}$ and the matrix elements $\left\langle f\left|O_{i}^{R}\right| b\right\rangle \equiv\left\langle f\left|O_{i}^{R}\right| b\right\rangle^{(0)}+g_{s}^{2} \cdot\left\langle f\left|O_{i}^{R}\right| b\right\rangle^{(1)}+O\left(g_{s}^{4}\right)$ are needed to order $g_{s}^{2}\left[g_{s}^{3}\right.$ for $\left.A^{\text {full }}=O\left(g_{s}\right)\right]$.

For the penguin operators, $O_{3} \ldots O_{6}$, which arise at $\mu=M_{W}$ only in the combination $O_{P}$, the NLL matching condition (33), combined with (28) and (32), yields

$$
c_{P}^{(1)}\left(M_{W}\right)=\frac{\alpha_{s}\left(M_{W}\right)}{4 \pi}\left[F_{1}\left(\frac{m_{c}^{2}}{M_{W}^{2}}\right)-F_{1}\left(\frac{m_{t}^{2}}{M_{W}^{2}}\right)+\frac{2}{3}\left(\xi_{\mathrm{R}}+\ln \frac{m_{c}^{2}}{M_{W}^{2}}\right)\right] .
$$

\footnotetext{
${ }^{10} m_{t}^{2} / M_{W}^{2}$ and consequently $\ell n m_{t}^{2} / M_{W}^{2}$ are considered as $O(1)$; this is consistent with the approximation that the top and the $W$ are integrated out at the same scale.
} 
Similarly, one determines the (scheme dependent) $O\left(\alpha_{s}\right)$ corrections to the coefficients of $O_{1,2}$ (see ref. [20]). Note that the logarithms in (30) and (34) combine with $\Delta F_{1}\left(\frac{q^{2}}{m_{i}^{2}}\right)$

and $F_{1}\left(\frac{m_{i}^{2}}{M_{W}^{2}}\right)$, respectively $(i=u, c)$. Therefore, $\left\langle s q \bar{q}\left|O_{2}^{R}\right| b\right\rangle^{(1)}$ and $c_{P}^{(1)}\left(M_{W}\right)$ remain finite in the limit of negligible internal quark masses $\left(m_{i} \rightarrow 0\right)$.

Since $O_{g}$ and $O_{\gamma}$ do not mix into the four-quark operators $O_{1} \ldots O_{6}$, one can consistently restrict a NLL analysis to this subset [20]. At $\mu \neq M_{W}$, the $O\left(\alpha_{s} \cdot 1\right)$ scheme dependence entering via the initial values of the coefficients [due to the renormalization of the one-loop matrix elements $\left\langle f\left|O_{i}^{R}\right| b\right\rangle^{(1)}$ in (33)] is canceled by the corresponding scheme dependence in $E^{(N L L)}$ (see Buras et al. 20 for a detailed analysis of this issue).

In decay modes, like $b \rightarrow s g, s \gamma, s g g, s g \gamma$, etc., where tree-level and one-loop matrix elements contribute at the same (leading) order in $g_{s}$, all divergencies in the one-loop matrix elements of the four-Fermi operators cancel[ $[$, and therefore, no renormalization scheme dependence remains even in LL approximation. However, a NLL analysis would be helpful to reduce the $\mu$-dependence $\square$ of the physical results, which is the more pronounced the larger the QCD corrections are relatively to the uncorrected results at $\mu=M_{W}$ (e.g. in $b \rightarrow s \gamma$ [31]). For the operators $O_{g}$ and $O_{\gamma}$, the $O\left(g_{s}^{3}\right)$ matching (33) requires the finite parts of all corresponding two-loop graphs in the full theory, and the $\gamma_{k l}^{(2)}$ for $k=1 \ldots 6$ and $l=g, \gamma$ have to be determined from tree-loop diagrams. So far, such a calculation has not yet been attempted and the mixing of the four-quark operators into $O_{g}\left(O_{\gamma}\right)$ is carried out only with LL times $g_{s}(e)$ precision [26].

\section{Discussion}

In this paper we have studied the validity of the equations of motion within the framework of an effective field theory described by a Lagrangian $\mathcal{L}_{\mathrm{o}}+\mathcal{L}_{\text {eff }}$, where the interactions from $\mathcal{L}_{\mathrm{o}}$ (e.g. QCD or QED) are to be treated at arbitrary order and at the loop level. We conclude that, in fact, $\mathcal{L}_{\text {eff }}$ can be rewritten by freely using the naive classical EOM (i.e. without gauge-fixing and ghost terms) derived from $\mathcal{L}_{\mathrm{o}}$, provided one considers only on-shell matrix elements at first order in $\mathcal{L}_{\text {eff }}$. In particular, the effective Lagrangian may be reduced by the EOM before (RG improved) short-distance QCD corrections are evaluated.

Although this result is not surprising, the reasoning involves non-trivial ingredients,

\footnotetext{
${ }^{11}$ In fact, this is readily seen with the help of the EOM: The counterterms for e.g. $\left\langle\operatorname{sgg}\left|O_{2}\right| b\right\rangle^{(1)}$ are proportional to the operator $P_{1}$; the latter is equivalent to $O_{P}$ by the EOM and contributes just to the renormalization of matrix elements like (30).

${ }^{12}$ By construction of the RG evolution, the $\mu$-dependence must cancel order by order in $\alpha_{s}$. However, the RG evolution of the coefficients includes all orders of $\alpha_{s}$ times log, while the matrix elements are evaluated only to order $g_{s}\left(g_{s}^{3}\right)$ in LL (NLL) approximation.
} 
which are in principle well-known in a general context [5, 7]. The essential steps have been carried out explicitly here: Besides renormalization effects for operators which vanish by the classical EOM, we have investigated the relevance of the additional terms in the correct EOM which arise from the gauge-fixing and ghosts. The possibility of dropping these terms is due to the fact that they appear in a combination which is the BRS variation of some other operator; such operators do not mix into physical operators during the $\mathrm{RG}$ evolution and their physical matrix elements vanish identically.

On the other hand, when the effective interactions from $\mathcal{L}_{\text {eff }}$ are to be used at higher order, operators which vanish by the classical EOM do indeed lead to physical effects. We have described an explicit procedure to derive a reduced effective Lagrangian which is on-shell equivalent to the original one also beyond the linear order in $\mathcal{L}_{\text {eff }}$. In this procedure an operator $O_{\mathrm{EOM}}$, which vanishes by the classical EOM, is replaced by suitable (physical) operators which account for all non-vanishing contact terms in Green's functions with multiple insertions of $O_{\mathrm{EOM}}$.

Since the renormalization group evolution preserves the relations due to the EOM, we note that, it is not necessary for the definition of an ('on-shell') effective theory to specify a "canonical form" [2] of the set of operators: Although the matching with on-shell amplitudes determines only a class of on-shell equivalent effective Lagrangians, each of them will yield the same physical results after the RG evolution to any other scale.

The ghost operator, $O_{\mathrm{FP}}$, has also been discussed by Grigjanis et al. [17. However, there, $O_{\mathrm{FP}}$ did not arise through the EOM but was introduced by replacing the 'penguin' operator $P_{1}$ (三O $O_{7}$ in the notation of ref. [17]) through a combination of $P_{1}$ and $O_{\mathrm{FP}}$. This ad hoc step was justified by gauge invariance arguments [17, 32]: Transversality is demanded for the sum of the one-loop diagrams that renormalize $O_{7}$, and $O_{\mathrm{FP}}$ is added in order to compensate for a diagram where the two gluons from $O_{7}$ close a loop by emitting one (external) gluon via the three-gluon vertex of QCD. This reasoning is not really compelling since only the combination $O_{\mathrm{gf}}+O_{\mathrm{FP}}$ is BRS invariant (see appendix A); in fact, the non-transversal terms in ref. 32 can be compensated by counterterms which contain no ghosts but rather vanish by the EOM for the quarks. Our discussion shows that $O_{\mathrm{FP}}$ arises in a natural way after the use of EOM (10) however, always in combination with $O_{\text {gf }}$.

The operator $O_{\text {gf }}$ was investigated by Eeg and Picek [33]. They claim that the $\not q_{\mu}$ piece of the penguin subdiagram gives rise to double-log terms in the mixing of $\mathrm{O}_{2}$ into $O_{\gamma}$, whereas such terms are not reproduced in the effective Lagrangian approach [19, [17. We recall that the $q q_{\mu}$ piece corresponds to the operator $O_{\text {gf }}$ and note that there exist no two-loop diagrams for $b \rightarrow s \gamma$ where $O_{\mathrm{FP}}$ contributes. Thus, our result 
concerning the absence of physical effects from $O_{\mathrm{gf}}+O_{\mathrm{FP}}$ implies that the $\not q q_{\mu}$ pieces, and the double-logs, in fact cancel for the sum of all diagrams at hand, see fig. 4. (Indeed, this can be readily verified by standard Ward-Takahashi identities for the gluon-quark vertex.)

Besides the striking advantage of the EOM for calculations of short-distance QCD corrections, we find the EOM to be particularly useful to keep track of the effective (penguin induced) couplings that depend logarithmically on the internal quark masses. (Recall that the weak GIM suppression of such terms is, for instance, responsible for the amazing fact that a three-body decay like $b \rightarrow s q \bar{q}$ has a larger branching ratio than the related two-body decay $b \rightarrow s g$.) In the effective 'penguin' Lagrangian, $\mathcal{L}_{\mathrm{P}}$, logarithmic coefficients appear only for the operators $P_{1}$ and $\tilde{P}_{1}$, which contain the gluon or photon field in the combination $D_{\mu} G_{a}^{\mu \nu}$ or $\partial_{\mu} F^{\mu \nu}$, respectively; via the EOM they are equivalent to four-Fermi operators. Consequently, working at lowest order in $G_{F}$ and $\alpha_{s}$, the amplitudes for any $b \rightarrow s$ transition, can receive $\ell n x$-terms $\left(x=m_{i}^{2} / M_{W}^{2}\right)$ only through contributions from the local four-Fermi operators $\bar{s} \gamma_{\mu} L \frac{\lambda_{a}}{2} b \cdot \bar{q} \gamma^{\mu} \frac{\lambda_{a}}{2} q$ or $\bar{s} \gamma_{\mu} L b \cdot \bar{\ell} \gamma^{\mu} \ell$. From this point of view, the crucial - but easily missed - cancellations of the $\ell n x$-terms in $b \rightarrow$ sgg etc. [1] become rather obvious.

The distinction between local and momentum dependent parts of the amplitudes, which is convenient for purely calculational reasons in the full theory, arises in a natural way in the effective theory: There, the two parts originate from tree-level and oneloop matrix elements of the effective Lagrangian, respectively, and receive in general different corrections when short-distance QCD effects are included. Some processes, like $b \rightarrow s q \bar{q}$, have contributions from $O(1)$ tree-level matrix elements (of local penguin operators) and from $O\left(\alpha_{s}\right)$ one-loop matrix elements (of usual four-Fermi operators). A systematic and unambiguous treatment of both kinds of matrix elements requires the use of the next-to-leading results for the QCD-corrections. If only leading-log QCD corrections are included, the real parts of the amplitudes can be recovered only up to renormalization scheme dependent constants (which, of course, do not affect the imaginary parts). Actually, taking the 'leading-log' approximation literally, one can (or should) drop the one-loop matrix elements for $b \rightarrow s q \bar{q}$ entirely, and consequently, all $\mathrm{CP}$ asymmetries become zero [15]. In other decay modes, like $b \rightarrow s \gamma, s g, s g \gamma$, etc., no renormalization scheme dependence remains even in LL approximation, but a NLL analysis would be desirable to reduce the $\mu$-dependence of the results.

\section{Acknowledgements}

I would like to thank D. Wyler and M. Misiak for their helpful collaboration in various stages of this work, and A. Ali, Ch. Greub, M. Lüscher, T. Mannel, M. Simonius and 
H. Spiesberger for valuable discussions and comments. During part of this work I enjoyed kind hospitality at the University of Zürich and support by the Swiss National Science Foundation. 


\section{Appendices}

\section{Appendix A: Gauge-fixing and ghosts}

In the background field formalism, the gauge field is decomposed into a background field $B_{\mu}^{a}$ and the 'quantum field' $A_{\mu}^{a}$. The Lagrangian derives from the usual Yang-Mills and matter Lagrangian by replacing the gauge-field by $B_{\mu}^{a}+A_{\mu}^{a}$. To fix the gauge, one adds

$$
\mathcal{L}_{\text {gf }}=-\frac{1}{2 \xi}\left(D_{\mu}^{a b}[B] A_{b}^{\mu}\right)^{2}
$$

where we define, for general $V_{\mu}^{a}$ and $\Phi^{a}$,

$$
D_{\mu}^{a b}[V] \Phi^{b} \equiv \partial_{\mu} \Phi^{a}+g f_{a b c} V_{\mu}^{b} \Phi^{c} .
$$

The resulting ghost Lagrangian is

$$
\mathcal{L}_{\mathrm{FP}}=\bar{\eta}_{a} D_{\mu}^{a b}[B] D_{b c}^{\mu}[B+A] \eta_{c}
$$

and the corresponding currents on the r.h.s. of (5) become

$$
\left(J_{\mathrm{gf}}\right)_{a}^{\nu}=-\frac{1}{\xi} D_{a b}^{\nu}[B] D_{b c}^{\mu}[B] A_{\mu}^{c}, \quad \text { and } \quad\left(J_{\mathrm{FP}}\right)_{a}^{\nu}=-g_{s} f_{a b c}\left(D_{b d}^{\nu}[B] \bar{\eta}_{d}\right) \eta_{c} .
$$

The theory is invariant under the following BRS transformations

$$
\begin{aligned}
\delta A_{\mu}^{a} & =D_{\mu}^{a b}[B+A] \eta_{b}, \\
\delta B_{\mu}^{a} & =0 \\
\delta \eta_{a} & =\frac{g}{2} f_{a b c} \eta_{b} \eta_{c}, \\
\delta \bar{\eta}_{a} & =-\frac{1}{\xi} D_{a b}^{\mu}[B] A_{\mu}^{b}, \\
\delta \Psi & =i g \frac{\lambda^{a}}{2} \Psi \eta_{a}, \\
\delta \bar{\Psi} & =-i g \bar{\Psi} \frac{\lambda^{a}}{2} \eta_{a} .
\end{aligned}
$$

For any $Q_{\mu}^{a}$, which transforms as $\delta Q_{\mu}^{a}=g f_{a b c} Q_{\mu}^{b} \eta_{c}$, the operator $Q_{\mu}^{a} \cdot\left(J_{\mathrm{gf}}+J_{\mathrm{FP}}\right)_{a}^{\mu}$ can be written as a BRS variation

$$
Q_{a}^{\mu} \cdot\left(J_{\mathrm{gf}}+J_{\mathrm{FP}}\right)_{\mu}^{a}=\delta\left(-Q_{a}^{\mu} \cdot D_{\mu}^{a b}[B] \bar{\eta}_{b}\right)
$$

Since the currents transform as

$$
\begin{aligned}
\delta\left(J_{\mathrm{FP}}\right)_{a}^{\mu} & =g f_{a b c}\left(J_{\mathrm{gf}}+J_{\mathrm{FP}}\right)_{b}^{\mu} \eta_{c} \\
\delta\left(J_{\mathrm{gf}}\right)_{a}^{\mu} & =-\frac{1}{\xi} D_{a b}^{\mu}[B] D_{b c}^{\nu}[B] D_{\nu}^{c d}[B+A] \eta_{d}
\end{aligned}
$$


the BRS variation of $Q_{\mu}^{a} \cdot\left(J_{\mathrm{gf}}+J_{\mathrm{FP}}\right)_{a}^{\mu}$ (and of $\left.J_{\mathrm{gf}}\right)$ vanishes upon using the EOM for the ghosts

$$
D_{a b}^{\nu}[B] D_{\nu}^{b c}[B+A] \eta_{c}=0
$$

To obtain the corresponding expressions in a usual covariant gauge, one simply sets $B_{\mu}^{a}=0$ everywhere in the above formulae.

\section{Appendix B: Renormalization at higher order in $\mathcal{L}_{\text {eff }}$}

The non-linear source renormalization (see ref. [6] for more details) provides a conceptually simple method for the renormalization of Green's functions with multiple insertions of composite operators. The generating functional

$$
W=\log \int \mathcal{D} \Phi \exp i \int\left(\mathcal{L}_{\mathrm{o}}+J_{0}^{B} \Phi+J_{i}^{B} O_{i}\right) d x,
$$

includes sources for the elementary fields and for all composite operators (including the unity-operator). The bare sources, $J_{i}^{B} \equiv S_{i}\left[J_{1}^{R}, \ldots\right]$, are general functions of the renormalized sources, $J_{i}^{R}(x)$, and their derivatives. Renormalized (respectively bare) Green's functions with insertions of the $O_{i}$ are given by functional derivatives of $W$ with respect to the renormalized (bare) sources.

The bare sources have an expansion

$$
S_{i}\left[J_{1}^{R}, \ldots\right]=\left(J_{l}^{R}+L_{\mathbf{m n}}^{j k l} \partial^{\mathbf{m}} J_{j}^{R} \partial^{\mathbf{n}} J_{k}^{R}+O\left(\left(J^{R}\right)^{3}\right)\right)\left[\mu^{\epsilon \mathbf{D}} \hat{Z}\right]_{l i},
$$

where the $L_{\mathbf{m}}^{j k l}$ are power series of poles in $\epsilon$ with residues that depend on the couplings of $\mathcal{L}_{\mathrm{o}}$ only; and $\partial^{\mathbf{m}}$ is a condensed notation for possible coordinate derivatives, e.g. $\partial^{0}=1, \partial^{\mu_{1} \mu_{2}}=\partial^{2} / \partial x_{\mu_{1}} \partial x_{\mu_{2}}$, etc. The diagonal matrix $\mu^{\epsilon \mathbf{D}}$ contains powers of $\mu^{\epsilon}$ which are needed to leave the mass dimensions of the $J_{i}^{R} \epsilon$-independent and to keep $\hat{Z}$ dimensionless. The matrix of renormalization constants, $Z \equiv \mu^{\epsilon \mathbf{D}} \hat{Z}$, is the same as in (113) and provides only the renormalization of Green's functions with a single insertion of an $O_{i}$.

To evaluate amplitudes at higher order in $\mathcal{L}_{\text {eff }} \equiv \sum g_{i}^{B} O_{i}$, only Green's functions integrated over the coordinates of the insertions of $\mathcal{L}_{\text {eff }}$ are needed. Therefore, the $g_{i}^{B}$ can be viewed as additional couplings in the action and their (bare) values follow from (B.1):

$$
g_{i}^{B} \equiv G_{i}\left(g_{1}^{R}, \ldots, \mu\right)=S_{i}\left[g_{1}^{R}, \ldots\right]
$$

Since the $g_{i}^{R}$ are coordinate independent, only the non-derivative terms $(\mathbf{m}=\mathbf{n}=0)$ from eq. (B.1) contribute in $G_{i}$. The $\mu$-dependence of the $g_{i}^{R}$ is governed by the beta functions

$$
\left.\beta_{i} \equiv \mu \frac{d g_{i}^{R}}{d \mu}\right|_{g_{j}^{B}=\text { const. }}=-\left[\frac{\partial G}{\partial g^{R}}\right]_{i j}^{-1} \mu \frac{\partial G_{j}}{\partial \mu}
$$


On the other hand, the RG equation for the (generating functional of) the Green's functions

$$
\left(\mu \frac{\partial}{\partial \mu}+\beta \frac{\partial}{\partial g_{o}^{R}}+\gamma J_{0}^{R} \frac{\delta}{\delta J_{0}^{R}}+\gamma_{i}\left[J_{1}^{R}, \ldots\right] \frac{\delta}{\delta J_{i}^{R}}\right) W=0,
$$

requires also the knowledge of the derivative terms of eq. (B.1) for the 'anomalous dimension functions'

$$
\gamma_{i}\left[J_{1}^{R}(x), \ldots\right] \equiv \mu \frac{d}{d \mu} S_{i}\left[J_{1}^{R}(x), \ldots\right] .
$$

Of course, at first order in the $g_{i}^{R}$, the beta functions of the effective couplings are related to the anomalous dimension matrix (14),

$$
\beta_{i}=-\gamma_{i j} g_{j}^{R}+O\left(\left(g^{R}\right)^{2}\right)
$$

and the $\mu$-dependence of the "coefficients functions", $c_{i}(\mu)$, of eq. (15) is equivalent to the running of the effective couplings, $\bar{g}_{i}=c_{i}(\mu)+O\left(\left(g^{R}\right)^{2}\right)$, governed by the RG equation

$$
\left(\mu \frac{\partial}{\partial \mu}+\beta \frac{\partial}{\partial g_{o}^{R}}+\beta_{i} \frac{\partial}{\partial g_{i}^{R}}\right) \bar{g}_{j}\left(\mu, g_{o}^{R}, g_{1}^{R}, \ldots\right)=0 .
$$

\section{Appendix C: Penguin operators}

The operators $P_{1} \ldots P_{8 L}$, as defined in (21), are linearly independent when ignoring relations due to the EOM. In fact, together with the analogous operators having opposite chirality and with the various four-quark operators, they form a complete set of gauge invariant dimension five and six operators for hadronic $b \rightarrow s$ transitions.

The linear independence is readily verified from the Feynman-rules, which we shall list here for the $b(k) \rightarrow s(k)$ self-energy, written as $\frac{i}{4 \pi^{2}} \frac{G_{F}}{\sqrt{2}} \Sigma(k)$, and for the 1PI vertex for $b(p) \rightarrow s\left(p^{\prime}\right)+g\left(\epsilon_{a}^{\mu}, q\right)$, which we write as $\frac{i g_{s}}{4 \pi^{2}} \frac{G_{F}}{\sqrt{2}} \Gamma_{\mu}\left(p, p^{\prime}, q\right) \frac{\lambda^{a}}{2} \epsilon_{a}^{\mu}$. The contributions to $\Sigma$ and $\Gamma_{\mu}$ from the various operators are:

\begin{tabular}{c|c|c}
$P_{i}$ & $\Sigma(k)$ & $\Gamma_{\mu}\left(p, p^{\prime}, q\right)$ \\
\hline$P_{1}$ & 0 & $\left(-q^{2} \gamma_{\mu}+\not q_{\mu}\right) L$ \\
$P_{2}$ & 0 & $-2 i \sigma_{\mu \nu} q^{\nu}\left(m_{b} R+m_{s} L\right)$ \\
$P_{3}$ & 0 & $2\left[\left(p^{2}+p^{2}\right) \gamma_{\mu}-\not p p_{\mu}-\not p p_{\mu}^{\prime}-\not p^{\prime} p_{\mu}-\not p^{\prime} p_{\mu}^{\prime}+2 \not p^{\prime} \gamma_{\mu} \not p\right] L$ \\
$P_{4}$ & 0 & $2\left[\left(-p^{2}+p^{\prime 2}\right) \gamma_{\mu}+\not p p_{\mu}+\not p p_{\mu}^{\prime}-\not p^{\prime} p_{\mu}-\not p^{\prime} p_{\mu}^{\prime}\right] L$ \\
$P_{5}$ & $-k^{2} \not k L$ & $\left(-p^{2} \gamma_{\mu}-p^{\prime 2} \gamma_{\mu}-\not p^{\prime} \gamma_{\mu} \not p\right) L$ \\
$P_{6}$ & $-k^{2}\left(m_{b} R+m_{s} L\right)$ & $\left(-\gamma_{\mu} \not p-\not p^{\prime} \gamma_{\mu}\right)\left(m_{b} R+m_{s} L\right)$ \\
$P_{7 R}$ & $M_{W}^{2} \not k R$ & $M_{W}^{2} \gamma_{\mu} R$ \\
$P_{8 R}$ & $M_{W}^{2} m_{b} R$ & 0 \\
\hline
\end{tabular}


The form factors, which enter in a general decomposition of $\Sigma$ and $\Gamma_{\mu}$,

$$
\begin{aligned}
\Sigma(k)= & \left(c_{0} M_{W}^{2}+c_{1} k^{2}\right) m_{s} L+\left(d_{0} M_{W}^{2}+d_{1} k^{2}\right) m_{b} R \\
& +\left(e_{0} M_{W}^{2}+e_{1} k^{2}\right) \not k L+\left(f_{0} M_{W}^{2}+f_{1} k^{2}\right) \not k R, \\
\Gamma_{\mu}\left(p, p^{\prime}, q\right)= & {\left[\left(g_{00} M_{W}^{2}+g_{01} q^{2}+g_{02} p^{2}+g_{03} p^{\prime 2}\right) \gamma_{\mu}\right.} \\
& \left.+g_{1} \not p p_{\mu}+g_{2} \not p p_{\mu}^{\prime}+g_{3} \not p^{\prime} p_{\mu}+g_{4} \not p^{\prime} p_{\mu}^{\prime}+g_{5} \not p^{\prime} \gamma_{\mu} \not p\right] \cdot L \\
& +\left(g_{6} \gamma_{\mu} \not p+g_{7} \not p^{\prime} \gamma_{\mu}+g_{8} p_{\mu}+g_{9} p_{\mu}^{\prime}\right) \cdot\left(m_{b} R+m_{s} L\right) \\
& +h_{0} M_{W}^{2} \gamma_{\mu} R,
\end{aligned}
$$

can be found in ref. [11]. They satisfy relations due to Ward-Takahashi identities,

$$
\begin{aligned}
& g_{00}=e_{0}, \quad h_{0}=f_{0}, \\
& c_{1}=d_{1}=g_{7}+g_{9}, \quad e_{1}=g_{01}+g_{02}+g_{1}, \quad f_{1}=0, \\
& g_{01}=\frac{1}{2}\left(g_{2}-g_{1}\right), \quad g_{02}=\frac{1}{2}\left(g_{2}+g_{1}\right)+g_{5},
\end{aligned}
$$

and due to the symmetry in $p$ and $p^{\prime}$ (up to order $m_{b, s}^{2} / M_{W}^{2}$ ),

$$
g_{03}=g_{02}, \quad g_{3}=g_{2}, \quad g_{4}=g_{1}, \quad g_{7}=g_{6}, \quad g_{9}=g_{8}
$$

The remaining form factors are in one-to-one correspondence with the coefficient functions $c_{i}^{\prime}$ of the operators $P_{i}$

$$
\begin{array}{llll}
c_{1}^{\prime}=\frac{1}{2}\left(g_{2}-g_{1}\right), & c_{2}^{\prime}=-\frac{1}{2} g_{8}, & c_{3}^{\prime}=-\frac{1}{4}\left(g_{1}+g_{2}\right), & \\
c_{4}^{\prime}=0, & c_{5}^{\prime}=g_{1}+g_{2}+g_{5}, & c_{6}^{\prime}=g_{6}+g_{8}, \\
c_{7 L}^{\prime}=-e_{0}, & c_{7 R}^{\prime}=-f_{0}, & c_{8 L}^{\prime}=-c_{0}, & c_{8 R}^{\prime}=-d_{0} .
\end{array}
$$

The relations for photonic operators are similar: The 1PI vertex for $b(p) \rightarrow s\left(p^{\prime}\right)+$ $\gamma\left(\epsilon_{\mu}, q\right)$, written in the form $\frac{i e}{4 \pi^{2}} \frac{G_{F}}{\sqrt{2}} \tilde{\Gamma}_{\mu}\left(p, p^{\prime}, q\right) \epsilon^{\mu}$, vanishes for $P_{1} \ldots P_{4}$, while $\tilde{\Gamma}_{\mu}=\Gamma_{\mu}$ for $\tilde{P}_{1} \ldots \tilde{P}_{4}$ and $\tilde{\Gamma}_{\mu}=-\frac{1}{3} \Gamma_{\mu}$ for $P_{5} \ldots P_{8}$. The $\tilde{c}_{1}^{\prime} \ldots \tilde{c}_{4}^{\prime}$ are obtained by replacing $c_{i}^{\prime}$ and $g_{j}$ in (C.4) by $\tilde{c}_{i}^{\prime}$ and $\tilde{g}_{j}$, where the latter are also given in [1].

\section{Appendix D: Inami-Lim functions}

For on-shell quarks (but off-shell gluon) the $\bar{s} b g$-vertex has only two independent form factors, the Inami-Lim functions $F_{1}$ and $F_{2}$ [27:

$$
\Gamma_{\mu}\left(p, p^{\prime}, q\right)=F_{1}(x)\left(q^{2} \gamma_{\mu}-q_{\mu} q\right) L-F_{2}(x) i \sigma_{\mu \nu} q^{\nu}\left(m_{b} R+m_{s} L\right) .
$$

After applying the EOM to the effective vertices in $\mathcal{L}_{\mathrm{P}}$, all coefficients can be expressed in terms of $F_{1}$ and $F_{2}$; their explicit expressions and the limits for $x \longrightarrow 0$ and $x \longrightarrow 1$ 
are as follows $\left(\eta \equiv \frac{1}{x-1}\right)$ :

$$
\begin{aligned}
F_{1}(x) & =\frac{1}{12}-\frac{1}{2} \eta^{3}+\frac{7}{12} \eta^{2}+\frac{7}{6} \eta+\left(\frac{1}{2} \eta^{4}-\frac{1}{3} \eta^{3}-\frac{3}{2} \eta^{2}\right) \ln x \\
& \longrightarrow-\frac{2}{3} \ln x+\frac{16}{3} x \ln x+\frac{3}{2} x \quad(x \longrightarrow 0) \\
& \longrightarrow \frac{43}{72}-\frac{19}{60}(x-1) \quad(x \longrightarrow 1) \\
F_{2}(x) & =-\frac{3}{2} \eta^{3}-\frac{9}{4} \eta^{2}-\frac{1}{2} \eta+\frac{1}{4}+\left(\frac{3}{2} \eta^{4}+3 \eta^{3}+\frac{3}{2} \eta^{2}\right) \ln x \\
& \longrightarrow \frac{1}{2} x \quad(x \longrightarrow 0) \\
\longrightarrow & \frac{1}{8}+\frac{1}{20}(x-1) \quad(x \longrightarrow 1)
\end{aligned}
$$

For the $\bar{s} b \gamma$-vertex $F_{1}, F_{2}$ have to be replaced by $\tilde{F}_{1}, \tilde{F}_{2}$ in (D.1). The magnetic moment, $\tilde{F}_{2}$, is given by

$$
\begin{aligned}
\tilde{F}_{2}(x) & =\frac{1}{2} \eta^{3}+\frac{9}{4} \eta^{2}+\frac{29}{12} \eta+\frac{2}{3}+\left(-\frac{1}{2} \eta^{4}-\frac{5}{2} \eta^{3}-\frac{7}{2} \eta^{2}-\frac{3}{2} \eta\right) \ln x \\
& \longrightarrow \frac{7}{12} x \quad(x \longrightarrow 0) \\
& \longrightarrow \frac{5}{24}+\frac{13}{120}(x-1) \quad(x \longrightarrow 1)
\end{aligned}
$$

$\tilde{F}_{1}$ depends on the gauge in the electroweak sector, but combines — via the EOM — with the coefficients of the semi-leptonic four-Fermi operators to a gauge-invariant expression 27, 28.

The form factor of the momentum dependent part of the amplitude for $b \rightarrow s q \bar{q}$ [see (25), (30)] is given by

$$
\begin{aligned}
\Delta F_{1}(z) & =-4 \int_{0}^{1} u(1-u) \ln [1-z u(1-u)] d u \\
& =\frac{2}{3}\left\{\frac{5}{3}+\frac{4}{z}+\left(1+\frac{2}{z}\right) R(z)\right\},
\end{aligned}
$$

where, setting $r \equiv \sqrt{|1-4 / z|}$,

$$
R(z)= \begin{cases}r \ln \frac{r-1}{r+1} & (z<0) \\ -2+z / 6+z^{2} / 60+z^{3} / 420 \cdots & (z \rightarrow 0) \\ 2 r \arctan (r)-r \pi & (0<z<4) \\ r \ln \frac{1-r}{1+r}+i r \pi & (z>4)\end{cases}
$$




\section{References}

[1] E. Witten, Nucl. Phys. B122 (1977) 109;S. Weinberg, Phys. Lett. B91 (1980) 51;A. Cohen, H. Georgi, B. Grinstein, Nucl. Phys. B232 (1984) 61;H. Georgi, Nucl. Phys. B361 (1991) 339

[2] H. Georgi in ref. [1] and C. Arzt, University of Michigan, preprint UM-TH-92-28

[3] W. Zimmermann, in Lectures on Elementary Particles and Quantum Field Theory, Brandeis University Summer Institute, Cambridge, MIT Press 1970

[4] J. Collins, Nucl. Phys. B92 (1975) 477;P. Breitenlohner, D. Maison, Commun. math. Phys. 52 (1977) 11

[5] S. Joglekar, B.W. Lee, Ann. of Physics 97 (1976) 160; S. Joglekar, Ann. of Physics 108 (1977) 233; 109 (1977) 210; see also H. Kluberg-Stern, J.B. Zuber, Phys. Rev. D12 (1975) 482; ibid. 3159

[6] G.M. Shore, Nucl. Phys. B362 (1991) 85

[7] J. Collins: Renormalization, Cambridge, Cambridge University Press 1984)

[8] R. Ammar et al. (CLEO), CLNS-93-1212

[9] W.S. Hou, A. Soni, H. Steger, Phys. Rev. Lett. 59 (1987) 1521; W.S. Hou, Nucl. Phys. B308 (1988) 561; R. Grigjanis, P. O'Donnell, M. Sutherland, H. Navelet, Phys. Lett. B224 (1989) 209

[10] M. Bander, D. Silverman, A. Soni, Phys. Rev. Lett. 43 (1979) 242

[11] J. Liu, Y.-P. Yao, Phys. Rev. D41 (1990) 2147; H. Simma, D. Wyler, Nucl. Phys. B334 (1990) 283

[12] J.M. Gérard, W.S. Hou, Phys. Rev. Lett. 62 (1989) 855

[13] S. Bertolini, F. Borzumati, A. Masiero, Phys. Rev. Lett. 59 (1987) 180; N.G. Deshpande et al., ibid. p. 183

[14] H. Simma, D. Wyler, Phys. Lett. B272 (1991) 395

[15] R. Fleischer, TU Munich, preprint TUM-T31-26-92 (1992)

[16] G. Altarelli, R.K. Ellis, L. Maiani, R. Petronzio, Nucl. Phys. B88 (1975) 215 
[17] R. Grigjanis, P. O’Donnell, M. Sutherland, H. Navelet, Phys. Lett. B213 (1988) 355; Phys. Lett. B223 (1989) 239; Phys. Lett. B224 (1989) 209

[18] G. Cella, G. Curci, G. Ricciardi, A. Viceré, Phys. Lett. B248 (1990) 181;G. Cella, G. Ricciardi, A. Viceré, Phys. Lett. B258 (1991) 212.

[19] see ref. [13], and B. Grinstein, R. Springer, M. Wise, Phys. Lett. B202 (1988) 138; Nucl. Phys. B339 (1990) 269;B. Grinstein, M. Savage, M. Wise, Nucl. Phys. B319 (1989) 271

[20] F. Gilman, M. Wise, Phys. Rev. D20 (1979) 2392;A.J. Buras, M. Jamin, M.E. Lautenbacher, P. Weisz, Nucl. Phys. B370 (1992) 69; Nucl. Phys. B375 (1992) 501.M. Ciuchini, E. Franco, G. Martinelli, L. Reina hep-ph@xxx.lanl.gov 9212203

[21] H.D. Politzer, Nucl. Phys. B172 (1980) 349

[22] H. Simma, Ph.D. Thesis, Diss. ETH No. 9781, Swiss Federal Institute of Technology, Zürich, 1992

[23] P. Cho, B. Grinstein, Nucl. Phys. B365 (1991) 279

[24] K. Wilson, Phys. Rev. 179 (1969) 1499

[25] G. Altarelli, L. Maiani, Phys. Lett. B52 (1974) 351; M.K. Gaillard, B.W. Lee, Phys. Rev. Lett. 33 (1974) 108; M.A. Shifman, A.I. Vainshtein, V.I. Zakharov, Nucl. Phys. B120 (1977) 316; Phys. Rev. D18 (1978) 2583

[26] M. Misiak, Phys. Lett. B269 (1991) 161; Nucl. Phys. B393 (1993) 23

[27] T. Inami, C.S. Lim, Prog. Theor. Phys. 65 (1981) 297; 1772(E)

[28] G. Buchalla, A. Buras, M. Harlander, Nucl. Phys. B349 (1991) 1

[29] M. Tanimoto, Phys. Lett. B218 (1989) 481

[30] H. Simma, G. Eilam, D. Wyler, Nucl. Phys. B352 (1991) 367; J.M. Gérard, W.S. Hou, Phys. Rev. D43 (1991) 2909

[31] A. Ali, C. Greub, DESY preprint DESY 93-065 (1993)

[32] M. Sutherland, Ph.D. Thesis, University of Toronto (1989)

[33] J.O. Eeg, I. Picek, Phys. Lett. B224 (1989) 205 


\section{Figure Captions}

Fig. 1: Subdiagrams with insertions of $O_{\mathrm{EOM}}$ of (9). The crossed blob $\otimes$ denotes $O_{0}$, and the hatched ones represent the two- and three-gluon pieces of $D_{\mu} G^{\mu \nu}$. The dots indicate further fields in $Q$.

Fig. 2: Diagrams which, in principle, may generate one-loop mixing of $O_{\mathrm{gf}}+O_{\mathrm{FP}}$ into four-quark operators, or to finite contributions to the matrix element for $b \rightarrow s q \bar{q}$.

Fig. 3: Diagrams for the mixing of $O_{\text {gf }} \sim \not q q_{\mu}$ into $O_{\gamma}$. The cross $\times$ indicates the various places where the gluon from $O_{\text {gf }}$ has to be attached to. 

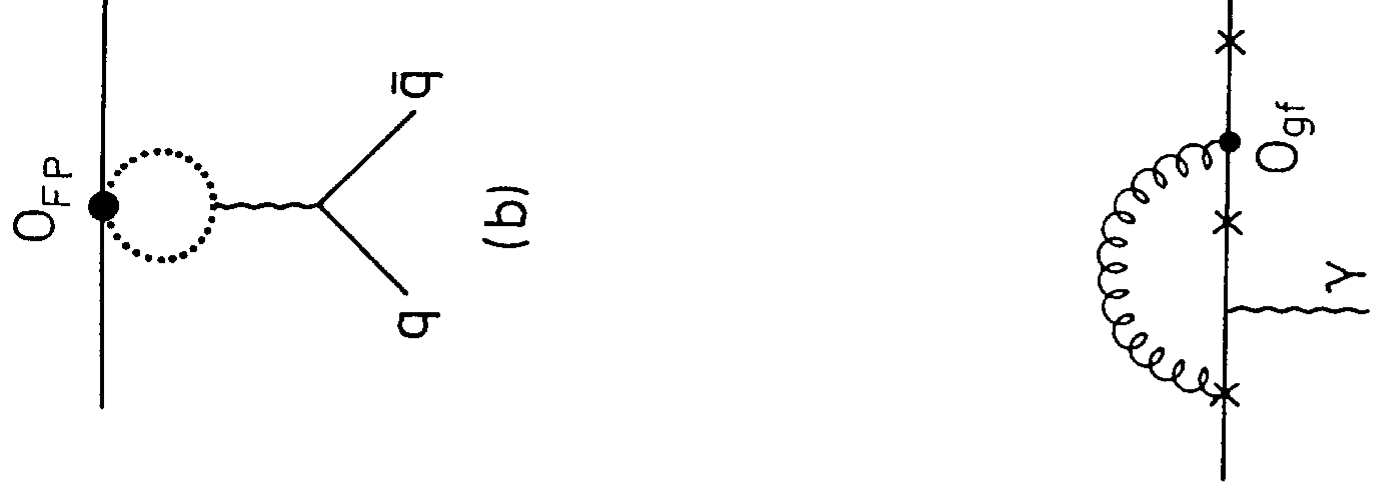

$\underset{\square}{\infty}$
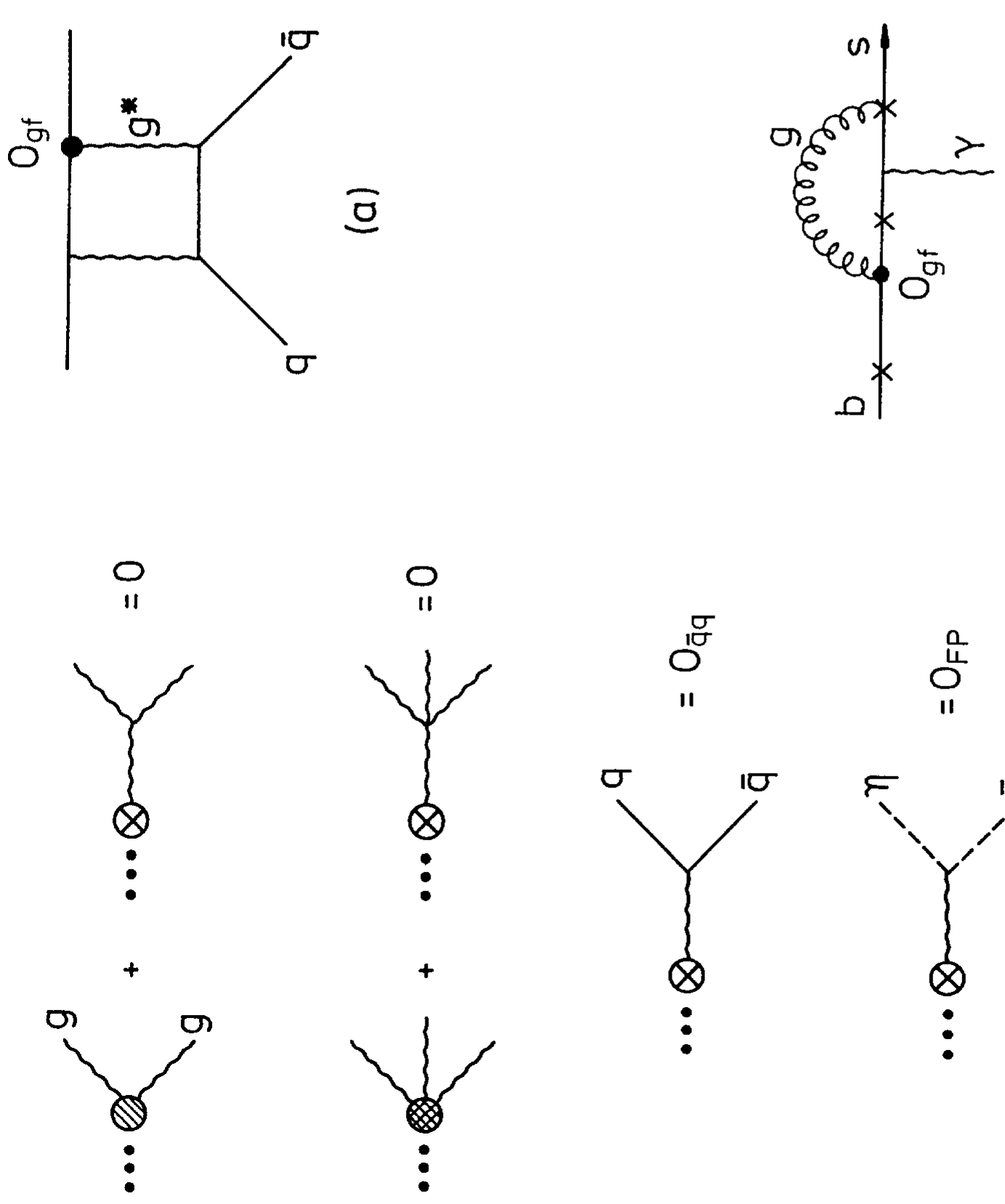

$\underset{\dot{a}}{\dot{\infty}}$ 